\title{
From Solute, Fluidic and Particulate Precursors to Complex Organizations of Matter
}

\author{
Ashit Rao*[a] and Helmut Cölfen*[b]
}

\begin{abstract}
The organization of matter from its constitutive units recruits intermediate states with distinctive degrees of self-association and molecular order. Existing as clusters, droplets, gels as well as amorphous and crystalline nanoparticles, these precursor forms have fundamental contributions towards the composition and structure of inorganic and organic architectures. In this personal account, we show that the transitions from atoms, molecules or ionic species to superstructures of higher order are intertwined with the interfaces and interactions of precursor and intermediate states. Structural organizations distributed across different length scales are explained by the multistep nature of nucleation and crystallization, which can be guided towards functional hybrid materials by the strategic application of additives, templates and reaction environments. Thus, the non-classical pathways for material formation and growth offer conceptual frameworks for elucidating, inducing and directing fascinating material organizations of biogenic and synthetic origins.
\end{abstract}

Keywords: Crystallization, material chemistry, mesocrystal, non-classical, superstructure

\section{Introduction}

Challenges in material science not only focus on the synthetic control over particle size and structure, but also strive towards the construction of complex materials with hierarchical organizations. Recent advances in the field of nucleation and crystallization inspire novel strategies for fabricating remarkable material architectures organized at different length scales. ${ }^{[1-3]}$ The related mechanistic pathways not only elucidate the emergence of non-equilibrium material morphologies and organizations, but also complement the inadequacies of classical notions. Rather than only focusing on the fundamental developments in the rapidly progressing field, this personal account systematically addresses the pathways towards complex material organizations from simple nanoscopic ingredients. We

\section{[a] A. Rao}

Freiburg Institute for Advanced Studies, Albert-Ludwigs-Universität Freiburg, Freiburg 79104, Germany

E-mail: Ashit.Rao@frias.uni-freiburg.de

[b] H. Cölfen

Physical Chemistry, Department of Chemistry, University of Konstanz, Konstanz 78464, Germany

E-mail: Helmut.Coelfen@uni-konstanz.de show that the multiplicity of interacting species can be effectively guided towards material forms with immense dynamic and structural complexity. With the growing importance of nanostructured hybrid materials in electrochemical, catalytic, magnetic and optical applications, the strategies of nucleation and crystallization that offer strategic control over functional material organizations are also discussed. ${ }^{[4,5]}$

For several materials, the nucleation and growth of a phase do not solely necessitate the unit-by-unit attachment of basic constituents, but also recruit material precursors with intermediate degrees of molecular organization relative to those exhibited by the basic building units and the corresponding bulk final material. The distinct molecular configurations, physical states and synergetic relations exhibited by these precursors greatly impact the pathways of nucleation and crystallization as well as the emergent architectures and morphologies. Even in a solid material, the constitutive (nano) particles present temporal evolutions of structural and crystallographic properties determined by their metastability, lattice energy and surface properties. ${ }^{[6]}$ This personal account shows that the sequential transitions involving atomic and molecular precursors, 


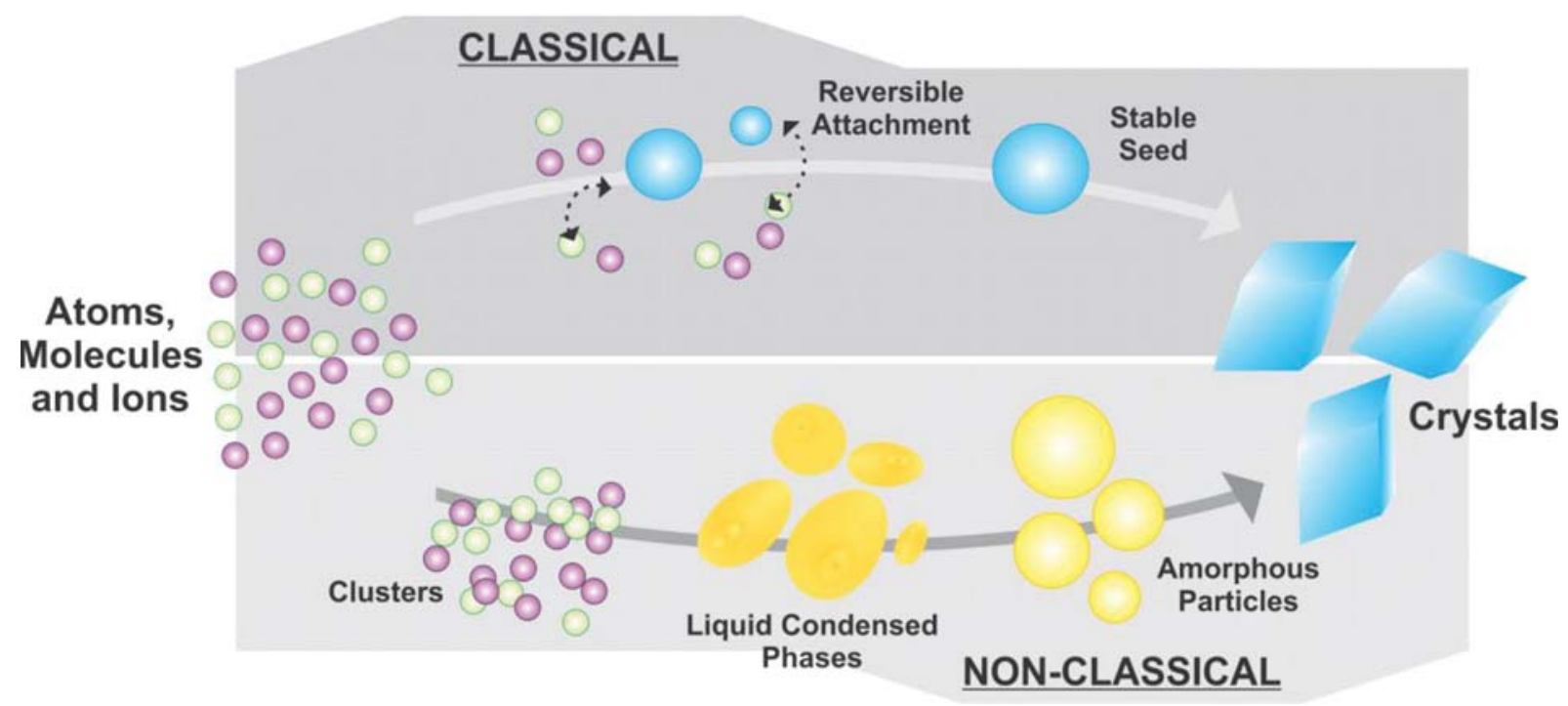

Figure 1. Following classical notions (top), the homogenous nucleation of particles involves the reversible attachment of basic building units to spherical nuclei, which can further grow on attaining critical radii. The critical radius is determined by the net free energy change, dependent on the surface energy per area and the bulk energy per volume of the forming phase. In relation, the non-classical routes (bottom) involve multistep formation and growth mechanisms with distinct contributions from cluster populations, liquid condensed phases and also mesoscale transformations involving amorphous and crystalline phases [Modified from ref. 8 with permission from American Association for the Advancement of Science].

solute clusters, fluidic intermediates, solid particles and organized nanostructured materials are based on their individual properties and synergistic interactions under influence of the microscopic and macroscopic environments.

\section{On the Nature of Material Precursors}

From emerging insights into the mechanisms of material nucleation, transformation and growth, a simple description of the stages of material genesis and ripening is provided (Figure 1). The regimes prior to the nucleation of solid 
particles can present soluble, dynamic entities consisting of primary building units termed as clusters, which exist in equilibrium with unassociated units. ${ }^{[7,8]}$ With increasing levels of supersaturation, an enhanced aggregation of unassociated units and clustered species, and also an accompanied loss of solvation lead to the phase separation of droplets via demixing or decomposition. ${ }^{[9]}$ In the absence of extrinsic stabilization, these metastable droplets can rapidly lose solvation and undergo densification, transforming to more stable amorphous and crystalline phases following Ostwald's step rule. These phase transitions can involve solid-state transformations and dissolution-precipitation reactions. Depending on the system energetics, these pathways can circumvent certain intermediates and also selectively favor one of several accessible pathways and products of nucleation and crystallization under thermodynamic and kinetic control. From this viewpoint, the authority of the non-classical nucleation and crystallization primarily originates from considerations towards (i) the physicochemical nature and the interactions between material precursors in form of solute clusters, droplets and nanoparticles, and also (ii) the synergistic effects of additives, templates and other reaction parameters on the interactions underlying material formation and growth. The physicochemical properties of precursor and intermediate states of matter not only illuminate the mechanistic emergence of complex forms and organizations, but also inspire novel synthetic strategies for directing hierarchical organizations. For these reasons, the chemical programming of self-assembly and structuration processes from dynamic nanoscopic units towards hierarchical material organizations necessitates fundamental descriptions of the nascent precursors and bridging intermediates.

\subsection{Solute Clusters}

Mechanistic contributions of cluster species are recognized for several metals, metal oxides and semiconductors such as $\mathrm{CdS}$, iridium, iron (oxyhydr) oxides, silver and titanium oxide particles. ${ }^{[10-17]}$ Conventionally addressed as 'nanoclusters', particles smaller than $2 \mathrm{~nm}$ typically exhibit atomic level rearrangements and distinct physical properties in relation to larger solid particles. ${ }^{[18-20]}$ This 'quasi-molten' state confers morphological and structural heterogeneity to cluster populations, as shown for gold nanoclusters. ${ }^{[21]}$ Hence the roughness of the free-energy landscape for the structural dynamics of cluster populations determines their stability, short-range order and in consequence their impact on the nucleation process (Figure 2). This is evident from other instances, such as the stability of certain CdS cluster species originating from the resistance of coordination structures towards deformation during agglomeration ${ }^{[10]}$ (Figure 2c). Even during the synthesis of semiconductor nanoribbons, the transient existence of $(\mathrm{CdSe})_{34}$ clusters prior to $(\mathrm{CdSe})_{13}$ clusters suggest defined configurations and stabilities of distinct nanocluster populations. ${ }^{[15,22]}$

Pre-nucleation clusters (PNCs) are thermodynamically stable populations of solute clusters. ${ }^{[23]}$ Despite the high configurational dynamism and solvation component, PNCs can exhibit distinct short-range order resembling emergent material structures even before the precipitation of solid particles. ${ }^{[24]}$ In this regard, both described as clusters of the basic building units viz. atoms, ions or molecules, PNCs can be considered as thermodynamically stable subsets of the entire ensemble of the nanocluster species. In the field of mineralization, the contributions of cluster species to nucleation events are reflected from relations between the thermodynamic stabilities of PNCs and the solubility products of the corresponding products. ${ }^{[7]}$ Recent accounts of short-range order in nanoscopic mineral precursors as small as $2 \mathrm{~nm}$ (corresponding to about $7 \mathrm{CaCO}_{3}$ units) raise fascinating questions about ingrained molecular configurations that enable proto-structuration reminiscent of the emergent solids. ${ }^{[25,26]}$ Of the potential factors that determine the configurational states of PNCs, the speciation and solvation characteristics of the basic building units are crucial. Molecular simulations relate the structural dynamism of cluster populations to the protonation states of building units $^{[27]}$ (Figure 2a). The solvation state of ions is an often overlooked factor, which turned out to be the driving force for the formation of $\mathrm{CaCO}_{3}$ PNCs and the further fate of the PNCs. ${ }^{[9,28]}$ External modulators of solvation also regulate the formation of PNCs from nanoscopic solute precursors. For instance, PNC-associated ions and organic molecules presenting higher hydration contents hinder the dehydration of the ionic species, thereby enhancing the supersaturation levels required for particle nucleation. ${ }^{[29,30]}$ Thus, the nucleation process cannot be deconstructed to a simple aggregationbased size evolution from individual ions to particles, but also is ingrained by the configurational and molecular nature of solute precursors in relation to their environment ${ }^{[10,31]}$ (Figure $2 \mathrm{a}-\mathrm{c})$. Given the prospects of configurational motifs propagating through the reaction co-ordinate prior to and after the nucleation event, the forces contributing to the clustering of ions, atoms and molecules are fundamentally and technologically relevant.

Even for certain organic systems, structural similarities are evident between the solute clusters and the emergent crystallization products. Analogous intermolecular hydrogen bonding and molecular distances are exhibited by clusters and crystal synthons of $p$-acetanisidide. ${ }^{[36]}$ For benzoic acid dissolved in toluene, hydrogen-bonded dimers stack into tetramers by aromatic interactions, presenting higher order interactions similar to the crystalline synthons. ${ }^{[37]}$ Prenucleation species are also identified for all amino acids and recently 
(a)

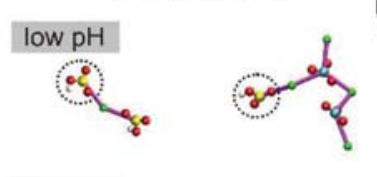

high $\mathrm{pH}$

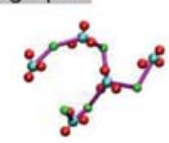

(d)

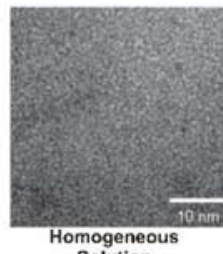

Solution

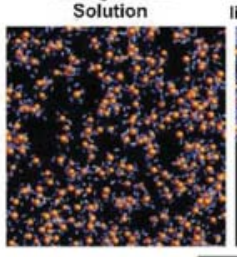

Spinodal decomposition (b)
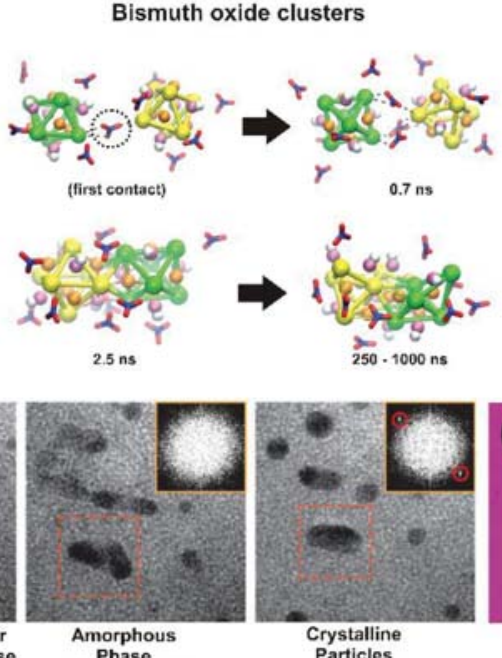

Amorphous
Phase
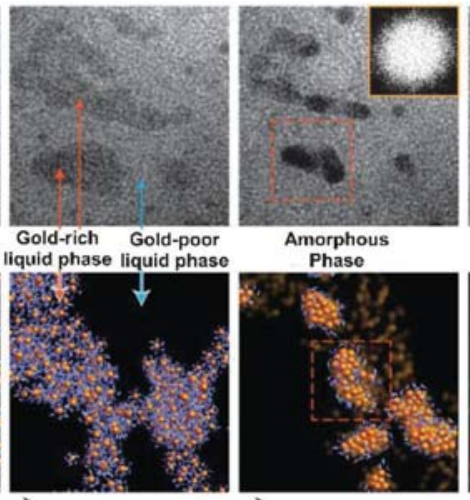

(r)
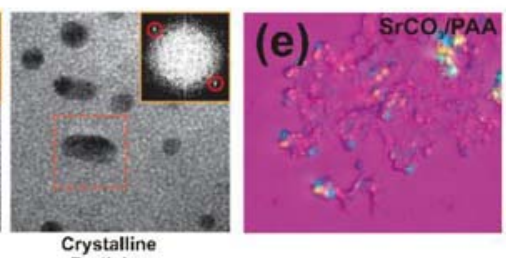

(c) $\mathrm{Cd}_{13} \mathrm{~S}_{4}(\mathrm{SH})_{10}$ Clusters

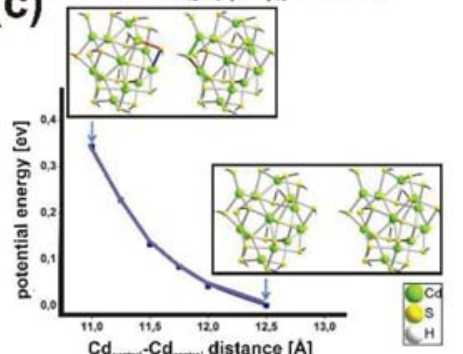

Particles
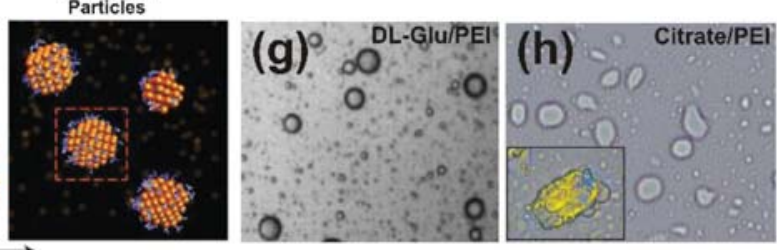

Figure 2. Mechanistic contributions of $(\mathrm{a}-\mathrm{c})$ solute clusters and $(\mathrm{d}-\mathrm{h})$ liquid condensed phases in nucleation and crystallization reactions. Computer simulations of (a) the termination effects of $\mathrm{HCO}_{3}{ }^{-}$ions (dotted circle) on the growth and stability of $\mathrm{CaCO}_{3}$ clusters, ${ }^{[27]}$ (b) the contributions of nitrate ions (dotted circle) as salt bridges between $\left[\mathrm{Bi}_{6} \mathrm{O}_{4}(\mathrm{OH})_{4}\right]\left(\mathrm{NO}_{3}\right)_{6}$ ] related cluster species, subsequently undergoing structural rearrangements and forming dimer species during bismuth oxide nucleation $^{[31]}$ and (c) $\mathrm{Cd}_{13} \mathrm{~S}_{4}(\mathrm{SH})_{18}$ PNCs exhibiting repulsive interactions based on thiol-stabilized surfaces and distinct coordination structures. ${ }^{[10]}$ (d) In situ observations of LCPs as precursors for amorphous and crystalline metal particles in supersaturated Au ${ }^{0}$ solutions. ${ }^{[32]}$ Polymer stabilized LCPs observed during the growth of $(\mathrm{e}, \mathrm{f})$ strontium carbonate crystals, ${ }^{[33]}(\mathrm{g})$ hierarchical microspheres of DL-glutamic acid ${ }^{[34]}$ and (h) irregular citrate microcrystals. ${ }^{[35]}$ [Reproduced with permissions from (a,d) Springer Nature, (b) John Wiley and Sons, (c) Royal Society of Chemistry, (e-g) American Chemical Society and (h) Springer Berlin Heidelberg].

for tartaric acid. ${ }^{[38,39]}$ With growing evidence for the conservation of molecular preferences during the progressions from clusters to nucleating particles, the configurational diffusion of solute clusters in the presence of soluble additives and complex interfaces requires attention. This knowledge can aid a 'faithful' propagation of configurational information from solute clusters to emergent materials, providing a better control over polymorph forms and structural organizations.

\subsection{Liquid Precursors}

Crystallization reactions for minerals, metals as well as organic molecules including proteins and other bioactive compounds can present liquid condensed phases (LCPs), represented by either droplets or gels ${ }^{[32,40-42]}$ (Figure 2). For minerals, Gower et al initially reported precursors with fluidic properties. ${ }^{[40]}$ The stabilization of a dense mineral LCP by miniscule amounts of anionic polymers led to a phase, termed as 'polymer induced liquid precursor (PILP) ${ }^{[33,40,43]}$ (Figure 2e, f). Detailed physicochemical investigations revealed that PILPs are in fact not polymer induced liquids but a polymer stabilized liquid phase, ${ }^{[44]}$ which could be formed by binodal decomposition of PNC aggregates ${ }^{[9]}$ (see below). The electrostatic and depletion forces contributing to polymer-mediated LCP stabilization confer unique mechanical properties to the fluidic mineral precursors. ${ }^{[40,45]}$ This is reflected by the distinct plastic and elastic modes of deformation as well as fluidic properties such as capillarity exhibited by mineral LCPs. For instance, the Young's modulus of $\mathrm{CaCO}_{3}$-based PILPs is estimated at about $2 \mathrm{MPa}$, similar to those of hydrogel materials. ${ }^{[42]}$

Detailed studies reveal that liquid-like droplets are transiently formed in course of mineral nucleation even without the requirement of external additives. With increasing supersaturation, PNCs are suggested to undergo condensation and desolvation, leading to the phase separation of transient mineral-enriched fluidic phases. ${ }^{[27,28,44,46]}$ Under reaction conditions that enable steady increases in supersaturation, a liquid-liquid binodal locus exists for the densification of PNC aggregates to liquid droplets. ${ }^{\left[{ }^{9]}\right.}$ However under conditions of high supersaturation, the emergence of mineral LCPs are suggested to occur via spinodal 
decomposition. ${ }^{[47,48]}$ In this scenario, a negligible energy barrier produces a spontaneous, diffusion-limited phase separation throughout the reaction media. ${ }^{[49]}$ With the identification of LCPs for several metal carbonates ${ }^{[33,50]}$ and also calcium phosphate, ${ }^{[51]}$ these fluidic precursors not only inspire synthetic avenues for hierarchical architectures but also offer novel insights towards physiological mineralization. ${ }^{[43,45,52]}$

Mechanisms of liquid-liquid demixing are also reported for the nucleation of metal and organic particles. The nucleation of gold and silver particles within the spinodal regime transiently produce two liquid phases, rich and deficient in the ionic precursors ${ }^{[32]}$ (Figure 2d). Amorphous particles form via the metal-rich liquid phase and then undergo crystallization. Contributions of gold-gold atom interactions and water-associated metastable gold complexes are suggested in these structural developments. Exemplifying organic materials (Figure $2 \mathrm{~g}, \mathrm{~h}$ ), the existence of LCP phases is demonstrated by PILPs composed of DL-glutamic acid or citrate stabilized by polyethyleneimine (PEI). ${ }^{[34,35]}$ Although the corresponding phase diagram identifies a small existence region of PILP phases relative to the thermodynamically stable crystalline forms, the long induction period for crystallization has enabled the characterization and manipulation of the fluidic material precursors. ${ }^{[34]}$ At present, an intense exploration of diverse LCP precursors is underway from fundamental perspectives of the formation and growth of diverse materials as well as with prospects of fabricating remarkable architectures, which are difficult to achieve via classical crystallization. This can even include industrial materials like pigments. ${ }^{[53]}$

For LCP-based nucleation schemes, the transitions from liquid precursors to solid amorphous or crystalline particles necessitate densification and desolvation. Due to a significant hydration component, the LCP droplets are generally larger than the corresponding solid products. Thus, in view of this multistep reaction, the definition of 'bottom-up' approaches towards particle formation and growth is not solely limited to a size evolution of building units, but also encompasses distinct progressions in the short-range order and solvation compositions from fluidic precursor to solid particles.

\subsection{Solid Particles: Crystalline and Amorphous Precursors}

Analogous to the mechanistic roles of atoms, ions and molecules in the classical descriptions of particle nucleation and growth, crystalline and amorphous particles can serve as modular building blocks for generating complex morphologies and superstructures via mesoscale transformations. ${ }^{[54-56]}$ Consider a hypothetical scenario wherein the colliding nanocrystals share identical crystallographic register prior to contact. Under a high driving force for crystallization, the particles can fuse establishing a larger particle with sharing crystallographic register. For an alternative situation in which the interacting nanoparticles are crystallographically misaligned, particle fusion can produce defects at the particleparticle interfaces such as grain boundaries and twinning. Yet experimental observations of interacting nanoparticles uncover remarkably complex mechanisms of material assembly and growth via the fusion of particles. For several materials including calcium carbonate, iron oxyhydroxide, titania and semiconductors, ${ }^{[57-60]}$ colloidal nanocrystals rotate and move in relation to neighboring partners until an optimal crystallographic co-register is attained. Only after relative co-alignment, the particles collide and merge via a mechanism termed as 'oriented attachment', which could be nicely visualized by liquid cell electron microscopy. ${ }^{[59]}$ Interestingly, early theoretical investigations revealed a strong driving force for the minimization of a grain misalignment as soon as this angle gets smaller than $15^{\circ},{ }^{[61]}$ which could be confirmed by experimental evidence. ${ }^{[62]}$

The initial co-alignment of nanocrystals in sufficient proximity appears to be primarily driven by Coulombic interactions; ${ }^{[63]}$ however for certain semiconductor nanoparticles, dipole-dipole interactions promote the stabilization of emergent superstructures ${ }^{[64]}$ besides the orientation of the nanocrystals prior to oriented attachment. ${ }^{[65]}$ In case of anisotropic nanocrystals, van der Waals interactions modulate inter-particle interactions, for instance contributing to the end-to-end or side-to-side modes of alignment for nano-rods and -plates. ${ }^{[6]}$ Thus, besides the considerable driving force emerging from the surface area minimization by crystallographic fusion of two nanoparticle interfaces, the mutual contributions from Coulombic, dipole-dipole and van der Waals interactions appear to generate the driving forces for the assembly, attachment and fusion of nanoparticles towards hierarchical superstructures.

Properties of composition, morphology, size, stability, structure and surface of the interacting particles regulate their alignment and fusion to superstructured materials (Figure 3). For example, the colloidal stabilization of synthetic nanocrystals is generally achieved by applying surface-active agents including alkyl chains and charged polymers. These modifications provide distinct surface energies and chemistries, which in consequence enable distinct self-assembly patterns in relation to $\mathrm{pH}$, temperature, solvent and redox environments. In certain instances, an optimal surface decoration enables particles to 'sense another' via oriented attachment and achieve relative co-orientation; concurrently the stabilizing shells produce short-range repulsive forces, which inhibit the fusion of nanocrystals and elimination of high-energy surfaces. This situation produces a superstructure composed of crystallographically aligned building units termed as a 


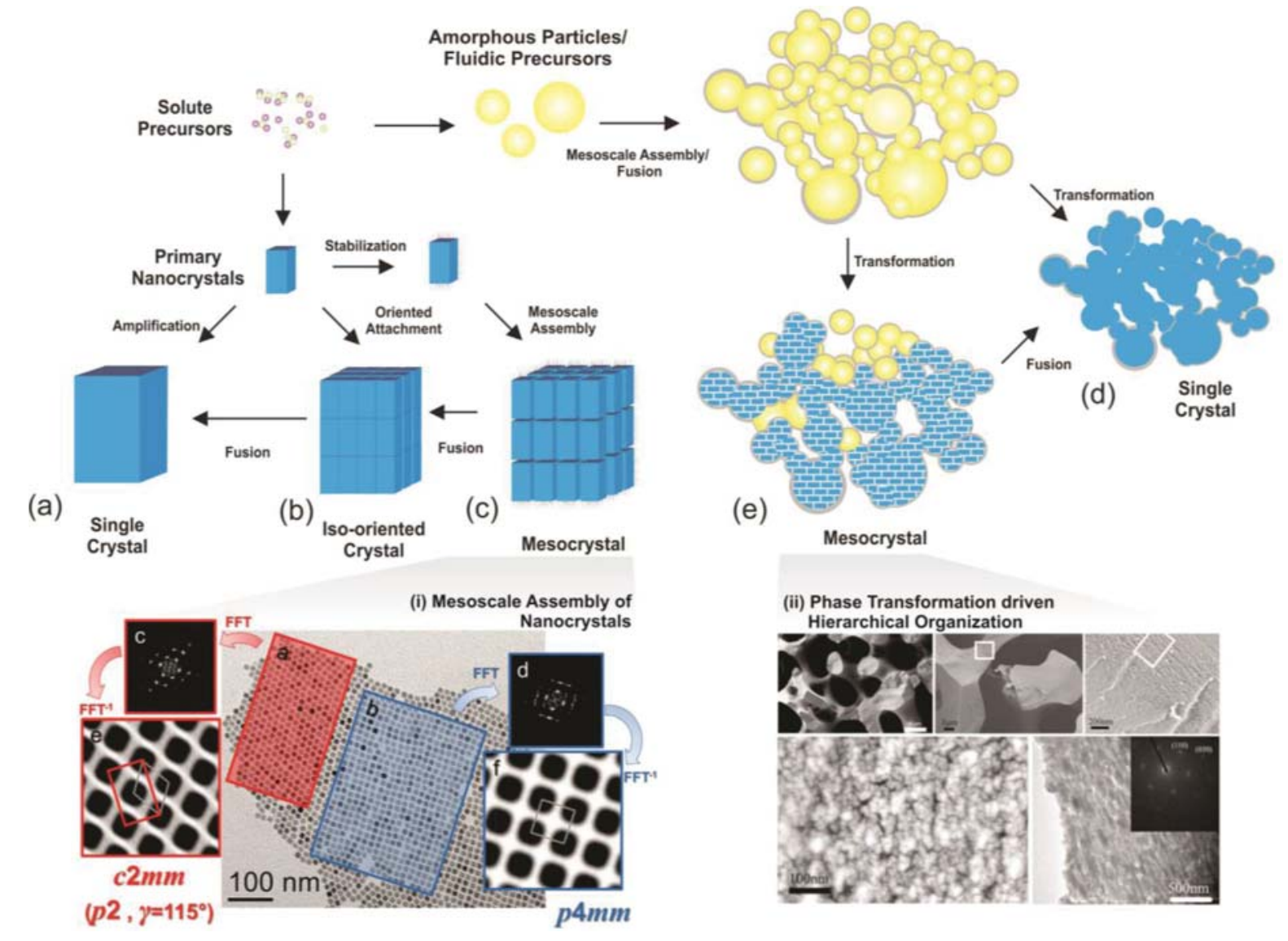

Figure 3. Schematic description of classical and non-classical crystallization mechanisms illustrating (a) classical growth of primary nanocrystals, (b) oriented attachment and fusion of nanocrystals towards an iso-oriented single crystal and (c) self-assembly of surface-stabilized primary particles forming a mesocrystalline organization. ${ }^{[55]}$ For amorphous precursors, an interplay between colloidal and phase stability leads to pathways of material formation that can produce (d) single (pseudomorphic) crystals and (e) mesocrystals, exhibiting intricate nano- and macro-features. ${ }^{[77]}$ Note the kinetic stability of mesocrystals and its relation with the transient fusion of constitutive nanocrystals towards single crystals. ${ }^{[78]}$ (i) Mesocrystals formed via particle self-assembly are exemplified by monolayers of magnetite nanocrystals made of coexisting domains with $c 2 \mathrm{~mm}$ and $p 4 \mathrm{~mm}$ symmetries. ${ }^{[79]}$ (ii) Mesocrystals derived from additive-controlled phase transformations are illustrated by the hierarchical structures of the sea urchin spine, comprised of space filling, co-oriented Mg-calcite nanocrystals embedded in an amorphous matrix. ${ }^{[75]}$ [Reproduced with permissions from $(\mathrm{a}-\mathrm{c})$ Royal Society of Chemistry, (d,e) Springer International Publishing, (i) John Wiley and Sons and (ii) National Academy of Sciences].

'mesocrystal' ${ }^{\text {[55,67-71] }}$ (Figure 3c). By definition, a mesocrystal is a nanostructured material with long-range order on the atomic scale, however constituted with individual nanoparticle units. ${ }^{[72]}$ These $2 \mathrm{D}$ or $3 \mathrm{D}$ crystalline superstructures enable micro/macroscopic materials to exhibit properties characteristic of nanocrystals, sometimes enhancing collective electronic and optical properties by near-field coupling. ${ }^{\text {[72,73] }}$ Note that the term 'mesocrystal' is strictly a structural definition and is not a superstructure exclusively derived from a stepwise attachment of nanocrystals. For example, mesocrystalline organizations can emerge via either transitions from dense, disordered particle clusters to ordered arrays or in certain biological instances (Figure 3e), from the phase transformation of amorphous precursors. ${ }^{[74-76]}$
In relation to the synthetic approaches for mesocrystals derived from the controlled assembly of nanoparticles, biological systems exercise a remarkable control over material form and (nano)structure by regulating the phase transformation of amorphous precursors ${ }^{[76,80,81]}$ (Figure $3 \mathrm{~d}$, e). Being disordered and isotropic, amorphous precursors produce atypical crystal morphologies and complex structures upon crystallization, distinct from the euhedral equilibrium counterparts predicted by Wulff's rule. ${ }^{[82]}$ The molecular mechanisms and kinetics of phase transformation determine the emergence of distinct structural organizations from amorphous precursors. ${ }^{[77,83,84]}$ For instance, the environments and other parameters of dissolution-reprecipitation reactions can produce a spectrum of crystalline architectures ranging from monoliths to polycrystals and also generate pseudomor- 
phic transformations and internal porosities. ${ }^{[83,84]}$ For material organizations derived from amorphous precursors, the mechanisms of structuration are guided by an interdependency between multiple factors including (i) (a) synchronous relations between dissolution and precipitation, (ii) relative solubility products of the reactants and products, (iii) local solvent conditions as well as (iv) ionic and molecular species regulating transformation kinetics and inter-particle interactions. An interplay between these factors is reflected in the mesocrystalline organization of the sea urchin spine, which is derived from the biogenic crystallization of an amorphous mineral precursor in the presence of ionic and (bio) polymeric additives. ${ }^{[6,75]}$ Combining the mechanisms of material formation by particle-attachment and phase transformation, crystal growth can proceed by the attachment of amorphous particles to crystalline surfaces. ${ }^{[3]}$ Analogous to the roles of atoms, molecular and ions in the Kossel model for layer-by-layer crystal growth, amorphous particles attach and organize on crystalline surfaces. ${ }^{[3]}$ Subsequent phase transitions can preserve the initial particle morphologies, resulting in granular crystal textures. Alternatively, the amorphous organization can undergo restructuration and particle fusion prior to transformation, producing smoother crystalline surfaces. The kinetic control of these colloidalepitaxy mechanisms produces distinct micro- and nanoorganizations. For instance, in polymer-containing or gel environments, the assembly, attachment and transformation of amorphous mineral particles produce porous and textured crystals. $^{[3,85]}$

Mesoscale transformations recruiting amorphous and crystalline units represent a powerful means to produce granular textures, anhedral morphologies and superstructured organizations. With the involvement of amorphous particles, the coupling of phase transformation with particle attachment and assembly often produces highly intricate pathways for material structuration. On account of low densities and slow sedimentation, the interactions between amorphous particles can be kinetically hindered, thus presenting a temporal window for synergistically guiding particle attachment and transformation. On the other hand, the diffuse interfaces, high solubility products and significant mobility of the constituents of amorphous particles increase the likelihood of attachment and fusion, in relation to crystalline counterparts. However, with a suitable application of ionic and polymeric additives, amorphous particles can be colloidally stabilized, also regulating the kinetics of crystallization. For instance, the nucleation of amorphous phases in the presence of certain polymers and stabilizing shells limits particle growth and coalescence. ${ }^{[25,86,87]}$ Alternatively certain small molecules and ions applied as isomorphic substituents impede the phase transformation of amorphous intermediates. ${ }^{[88]}$ In both approaches, the modes of action of the extrinsic constituents in phase stabilization invoke tendencies towards smaller particle sizes and higher hydration contents. This renders the simultaneous but independent control of the phase stability and colloidal properties of amorphous particles a challenging task in course of non-classical crystallization. Even with these physical boundaries, there are spectacular material organizations derived from amorphous particles that identify the cooperative relations between the phase stability and interparticle interactions as an important checkpoint for guiding crystallization reactions. ${ }^{[2,89,90]}$

\section{Pathways to Complex Organizations of Matter}

In view of the contributions of solutes, liquids, gels and particles in nucleation and crystallization, it is important to note that their transient existence and interactions with the developing material are not strictly exclusive. On account of unique physicochemical properties in terms of composition, morphology, size, solvation, stability and structure, the precursors and intermediates of materials can be selectively guided towards precise forms, morphologies and organizations by thermodynamic and kinetic influences. In addition, the manifestation of atomic, molecular and ionic building units as distinct precursors and intermediates reduces the driving force for crystallization, hence producing a reaction timescale responsive to external regulation. ${ }^{[1]}$ In addition, the introduction of the nascent material to defined interfaces and spaces can selectively enhance the transient stability of and also promote interactions between selective precursor, intermediate and product entities. Thus, the multistep nature of nucleation and crystallization is itself beneficial in providing avenues for the strategic guidance of emerging materials towards organizations and structures of interest. Note that the mechanisms discussed below are classified on basis of the primary precursor or intermediate impacting emergent form or organization. These schemes of classification and regulatory features are not always mutually exclusive and can collectively operate in producing complex morphological and structural outcomes.

\subsection{Nucleation Checkpoints}

Interactions between solute precursors and inorganic or organic additives can be broadly classified based on the mode of interaction. Firstly, certain additives capture clusters of atoms, molecules and ions, inhibiting further coalescence. This 'divide and protect' stabilization is illustrated by thiolate-protected gold clusters as well as 10,12pentacosadiynoic acid protected mineral clusters. ${ }^{[25,26,92]}$ Here the overall structure and energetics of nanoclusters are related to the chemical nature of the ligand and also the distinct arrangements of the core and the core-shell inter- 
(a)
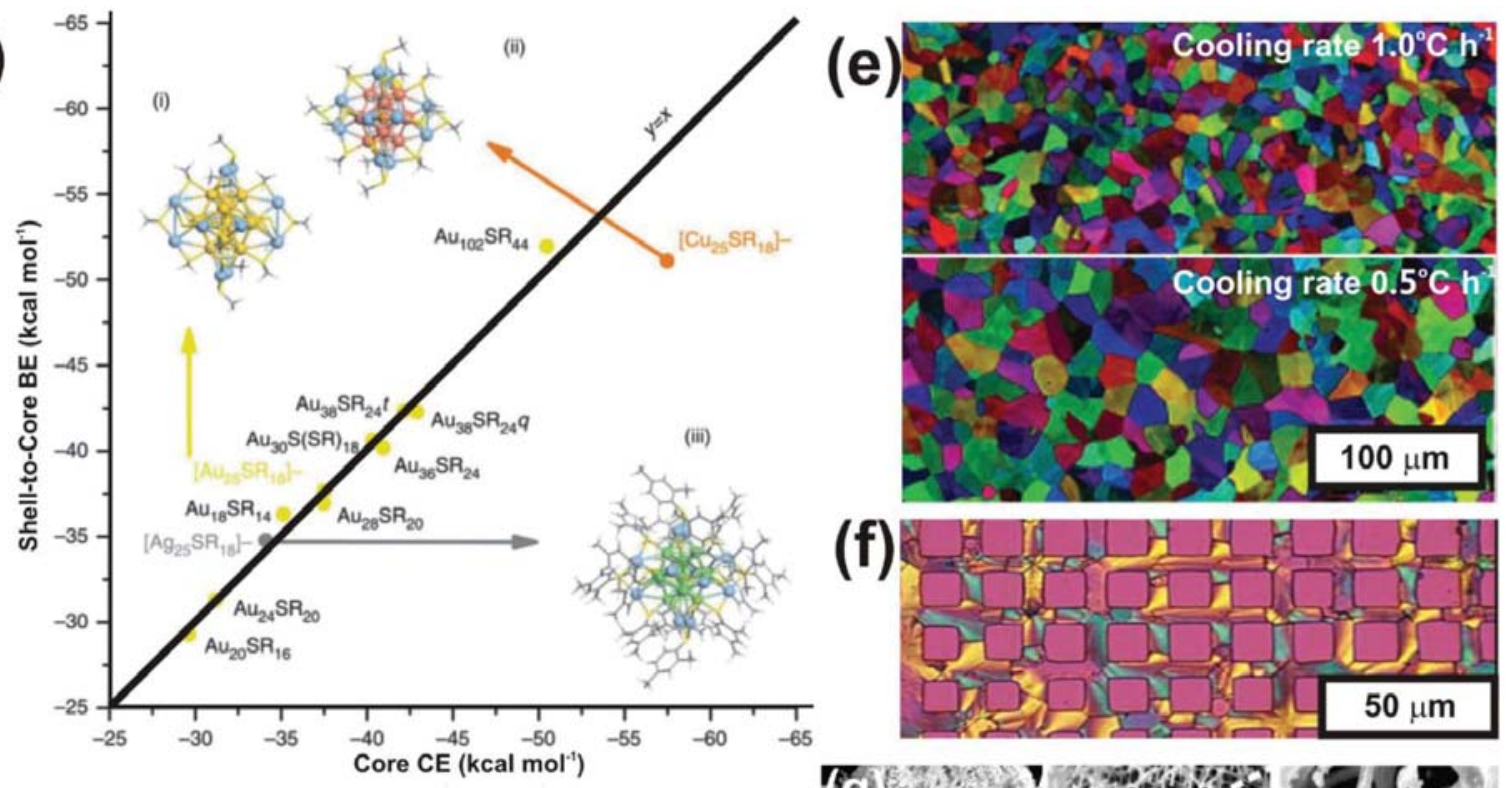

(f)

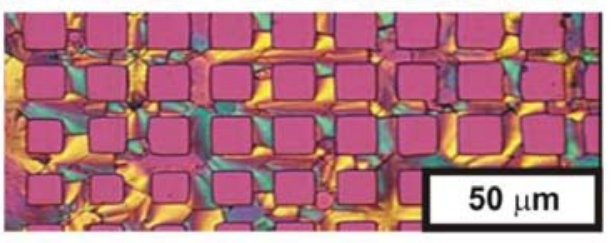

(b)

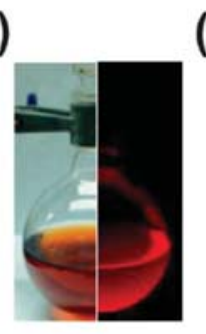

(c)

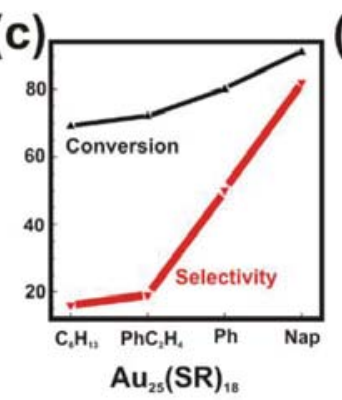

(d)

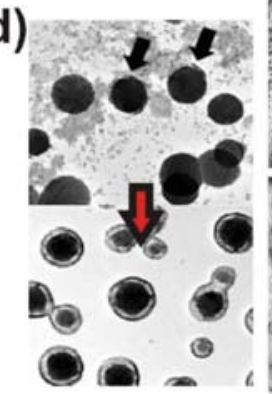

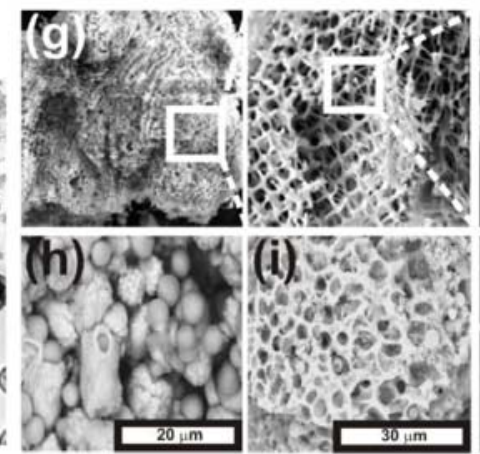

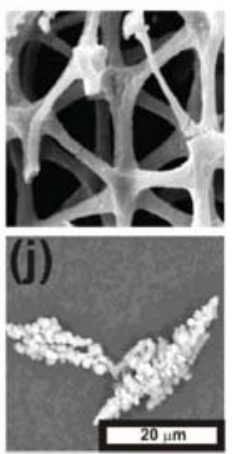

Figure 4. (a) Emergence of cluster stability illustrated from an apparent relation between the core cohesive energy (CE) of distinct thiolate-protected metal clusters and the corresponding shell to core binding energy $(\mathrm{BE}) .^{[93]}$ (b) Intense luminescence of a gold nanocluster species, ${ }^{[95]} \mathrm{Au}_{22}(\mathrm{SR})_{18} \cdot(\mathrm{c})$ Catalytic activity and selectivity of $\mathrm{Au}_{25}$ nanoclusters in Ullmann heterocoupling reactions influenced by the chemical nature of the protecting ligands. ${ }^{[96]}$ (d) Synthesis of hollow particles via the surface transformation of transient phytic acid-ion hybrid precursors to mineral shells. ${ }^{[98]}$ Control of material structure and organization by using fluidic precursors are illustrated by (e) the cooling rate dependent domain sizes of mesocrystalline mosaic films of DL-lysine, ${ }^{[102]}$ (f) self-assembled monolayerdirected patterns of $\mathrm{CaCO}_{3}$ crystals, ${ }^{[103]}(\mathrm{g})$ microporous crystals formed by the infiltration of inverse replica of sea urchin spines with liquid-like mineral precursors ${ }^{[104]}$ as well as composite particles of $(\mathrm{h})$ core-shell, (i) microporous sponge and (j) elongated morphologies formed during mineralization in the presence of PILPs based on (h, i) citrate/PEI and (j) caffeine/poly (acrylic acid) (PAA). ${ }^{[35]}$ [Reproduced with permission from (a) Springer Nature, (b-d,f, g) American Chemical Society, (e) John Wiley and Sons and $(\mathrm{h}-\mathrm{j})$ Springer Berlin Heidelberg]

face $^{[93]}$ (Figure 4a). Ligand-stabilized nanocluster catalysts and luminiscent gold clusters are instances of the 'divide and protect' approach applied towards functional materials with fine-tune physicochemical properties ${ }^{[94-96]}$ (Figure 4b, c). The reactivity of the stabilizing shell can also be precisely directed towards the self-assembly and chemical manipulation of the stabilized nanoclusters.

In the second approach, organic and inorganic solutes can interact and merge with building units forming hybrid clusters. With heterogeneous compositions, the solute clusters exhibit relatively distinct properties of stability, structure and solvation. For instance, poly-dentate ligands such as phytic acid and citric acid associate with ions and ion-clusters, producing polynuclear complexes. ${ }^{[97,98]}$ With increasing supersaturation levels, these hybrid complexes aggregate into larger assemblies and subsequently nucleate particles. Based on these interactions, the hybrid clusters can guide nucleation pathways towards particles of defined structures, polymorphs and sizes. For instance, in course of $\mathrm{CaCO}_{3}$ formation, phytic acid initially forms fluidic organic-inorganic complexes and subsequently produces hollow core-shell particles via interfacial ripening (Figure $4 \mathrm{~d}$ ). Even the incorporation of $\mathrm{Mg}^{2+}$ ions in $\mathrm{CaCO}_{3}$ clusters enhances the supersaturation levels required for mineral nucleation and also enables a relative stabilization of the nucleated amorphous mineral. ${ }^{[99]}$ 
With these chemical controls, the size as well as configurational and structural complexities of cluster species can be tuned within the ranges intermediate those of ions, atoms or molecules and solid particles, thereby providing a fine control over the composition, morphology and structure of nucleating particles. For example, with the strategic application of counter-ions and ligands, thermodynamically stable cluster populations can be synthesized to precisely regulate the synthesis of nanoparticles. ${ }^{[100,101]}$ Specifically, the short-range structures of nanoclusters are manipulated in order to synthesize particles with narrow size distributions and anisotropic structural features at high reaction yields. Hence in addition to the interesting catalytic, chiral, electronic and optical properties presented at the nanoscale, cluster populations offer a practical means to control the nanoparticle growth via non-classical nucleation.

\subsection{Liquid Precursors}

Although the metastability of LCPs can hinder applications in morphogenesis and structuration, a rational application of additives enables the transient stabilization and manipulation of fluidic precursors as PILPs. Following this approach, the contributions of fluidic material precursors are identified during the crystallization of diverse industrially-relevant organic compounds such as amino acids, drugs and pigments. ${ }^{[35,41,53,105]}$ For instance, a branched PEI stabilizes amorphous liquid droplets composed of DL-glutamic acid, whereas PAA enhances the stability of liquid precursors of basic amino acids, L-lysine and L-histidine in ethanol:water mixtures. ${ }^{[105]}$ Upon crystallization, the droplets yield microparticles, the porosity of which reflects crystallization mechanisms involving the demixing of solid crystals and a water miscible polymer-rich phase. ${ }^{[105]}$ Subsequent mechanistic studies on particle growth from PILPs identify the homogeneous nucleation of platelets and their radial orientation within the droplet environment, followed by dehydrationinduced shrinkage. ${ }^{[41]}$ The selective formation of nanoplates is attributed to the face-selective adsorption of PEI, driven by electrostatic interaction and hydrogen bonding. In generating fluidic material precursors, the liquid-liquid phase separation provides microenvironments for not only crystal nucleation and growth but also the assembly of nanocrystals following particle-based non-classical crystallization pathways. Due to the distinct microenvironment within the droplets, nucleation and crystallization reactions and also inter-particle interactions can encounter conditions of diffusion, $\mathrm{pH}$, polarity, supersaturation and viscosity, unlike those presented by the mother solution.

When LCPs are applied for controlling the organization of organic and inorganic materials, liquid-solid and liquidliquid interfaces are crucial factors. Recently, crystallization studies of D,L-glutamic acid identify the attachment of hydrated nanoscopic assemblies to pre-formed crystals, followed by the release of hydration water, structural relaxation and transformation to $2 \mathrm{D}$ crystals. ${ }^{[106]}$ However, on silica surface, the nanoscopic assemblies do not transform easily. Thus, initial wetting and (epitaxial) interactions with the solid surfaces contribute to the spatiotemporal regulation of material growth via liquid-like precursors. For mineral systems, the fluidic nature of PILPs enables an efficient production of intricate crystal architectures either by template-free or template-directed strategies. The template-free crystallization of mineral PILPs produces atypical crystal structures including cones, fibers, films and rods. ${ }^{[33,107,108]} \mathrm{On}$ the other hand, template-guided strategies for PILP crystallization offer better morphological control and also a remarkable structural integration with the template (Figure $4 \mathrm{f}, \mathrm{g}$ ). This is also evident from lamellar micro- and nano-structures derived from the mineralization of collagenous templates. Networks of the organic framework not only aid the infiltration of the scaffold by the fluidic mineral precursors, but also guide the organization of nanocrystals illustrated by [001] oriented hydroxyapatite particles. ${ }^{[52,109]}$

There are several instances of PILP-based crystallization strategies producing superstructured architectures such as mesocrystalline thin films and microspheres. For instance, mesocrystalline films produced via a PILP of D,L-lysine present five levels of structural hierarchy involving micro-patterns, fanshaped patterns, dendrites, fibers, lamellae, and nanoparticles, with a temperature-guided morphogenesis mechanism ${ }^{[102]}$ (Figure 4e). Inorganic mesocrystals produced via PILP intermediates are evident by calcite microparticles and $\mathrm{BaCO}_{3}$ fibres. ${ }^{[108,110]}$ With several reports on mesocrystalline structures emerging from PILPs, the suggested mechanism is based on the preferential incorporation or exclusion of additive species from the crystallization front. ${ }^{[45,77]}$ Supporting evidence comes from the release of mineral-associated polymer units during the dehydration and crystallization of PILP phases. ${ }^{[111,112]}$ Further investigations on the spatial distributions of LCP-bound molecules (i.e. occluded within the bulk material or expelled to the solvent) will aid the elucidation and control of complex material organizations.

Crystallization additives can also be applied as PILPs in order to regulate the growth and structure of composite materials. ${ }^{[35]}$ For instance, organic PILPs applied during mineralization produce composite particles of distinct form and structure (Figure $4 \mathrm{~h}-\mathrm{j}$ ). Mineralized in the presence of PILPs constituted of caffeine and an oppositely charged polymer, the solid hybrid particles are acicular, but morphologically distinct from the individual equilibrium shapes of $\mathrm{CaCO}_{3}$ and caffeine crystals. ${ }^{[35,113]}$ These observations suggest dynamic bi-directional interactions between the organic and inorganic constituents. The PILPs kinetically and spatially 
regulate the formation of the mineral phase, whereas the mineral species influence the physical properties of the organic PILP such as mechanics and stability. ${ }^{[35]}$ Thus, fluidic material precursors serve as spatiotemporal determinants of nucleation and crystallization during the synthesis of composite particles, in addition to their primary role as confined sources of material precursors.

\subsection{Controlled Assembly of Particles}

The construction of material superstructures via the assembly and integration of particulate units offers several advantages including efficient mass fluxes and high growth velocities. In comparison to ion-clusters and LCPs, the densities of solid particles are quite close to the final material. With significant contents of the fundamental precursors immobilized in a solid form, a better reaction control is accessible due to limited kinetic influences from diffusion, $\mathrm{pH}$ and osmotic conditions. Since the synthetic strategies for material fabrication via particle attachment generally involve two steps viz. (i) the nucleation and growth of primary particles and (ii) their mesoscale assembly and organization, the composition, shape and size dependent properties of particles can be initially optimized and subsequently transcribed into a bulk superstructured material. In this regard, the steps of fabrication can be independently optimized to achieve mesostructured materials with defined spatial organizations. For these benefits, the self-assembly of particles into ordered superstructures has drawn considerable attention.

During particle-directed material growth, the colloidal properties of interacting nanoparticles critically influence the emergent arrangements and superstructures. For instance, the volume fractions and also the distributions of morphology, size and shape of interacting particles can influence their collective behavior as fluidic, amorphous and crystalline phases. ${ }^{[14,115]}$ By tuning the composition, structure and interface of particles, the forces of particle alignment based on electrostatic and van der Waals, hard sphere repulsion as well as depletion interactions can be effectively guided towards complex patterns and packing organizations. ${ }^{[116]}$ For instance, the particle surfaces can be tailored with ligands of specific hydration, electrostatic, redox, hydrophobic and hydrophilic characteristics, which render inter-particle interactions responsive to external stimuli. In this regard, the assembly of crystalline and amorphous nanoparticles into structured materials can be directed by diverse alignment strategies based on (i) extrinsic or intrinsic physical fields, (ii) crystalline bridges, epitaxial growth and secondary nucleation, (iii) face selective binding molecules, (iv) spatial constraints and (v) an external matrix or template. ${ }^{[17,118]}$ Among the utilization of extrinsic fields to align nanocrystals, electric, magnetic and centrifugal forces are well explored (Figure 5a). For example, the growth mechanisms and structural features of mesocrystals derived from subunits of $\mathrm{Ag}_{2} \mathrm{O}$ nanoparticles are regulated using external electric fields. ${ }^{[119]}$ On the other hand, intrinsic electric dipoles guide the alignment of fluoroapatite nanoparticles in gelatin matrices, which present structural developments from hexagonal prismatic rods to dumbbells, eventually forming spherical morphologies. ${ }^{[120]}$ During the assembly of maghemite nanocubes, external magnetic fields control the translational and orientational order of the superlattices. ${ }^{[121,122]}$ Control over crystallization is also achieved via centrifugal fields applied to amorphous precursors. For instance, the stabilization of amorphous copper hydroxide acetate is initially achieved by using amphiphilic block copolymers. Under centrifugal fields, the concurrent assembly and transformation of the amorphous precursors produce highly condensed crystalline plates with tunable magnetic properties. ${ }^{[123]}$ The layering and mutual alignment of vanadium pentoxide particles toward mesocrystalline architectures is also demonstrated by ultracentrifugation. ${ }^{[124]}$ As shown for a bulk nacre-like synthetic composite, even mechanical pressure can achieve the co-orientation of constitutive particles under optimal conditions. ${ }^{[125]}$ Thus, based on the physical features of particulate units in terms of composition, size, structure, shape and stability, an appropriate extrinsic or intrinsic physical field can generate complex material organizations with intricate $2 \mathrm{D}$ and $3 \mathrm{D}$ patterns.

In the second approach (Figure 5b), certain synthetic and biogenic mesostructured materials exhibit constitutive particles interconnected by nano-bridges. ${ }^{[126,127]}$ Indicative of partial oriented-attachment, these bridges enable an effective propagation of crystallographic information through the material volume, simultaneously retaining granular surfaces and interiors. For biominerals, the occurrence of bridges is connected to the accumulation of organic and inorganic constituents in inter-crystalline spaces, ${ }^{[5,126,128]}$ which hinder the complete fusion of the crystalline units. Complex material superstructures also originate from secondary and epitaxial nucleation events. The contributions of secondary nucleation in material structuration are evident from hetero-structured microspheres consisting of calcitic equatorial loops and

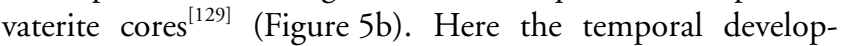
ments of distinct spatial patterns within the mineral particles reflect a potent spatiotemporal regulation of secondary nucleation achieved via the combined application of additives. Topochemical transformations also facilitate superstructured crystals, as evident by strontium titanate mesocrystals formed via single crystal anatase and $\mathrm{SrTiO}_{3}$ mesocrystals grown from $\mathrm{TiO}_{2}$ mesocrystals under hydrothermal conditions ${ }^{[130,131]}$ (Figure 5c). Additives preferentially binding to crystal faces also govern particle co-alignment and selfassociation. In other words, interfacial properties are engi- 


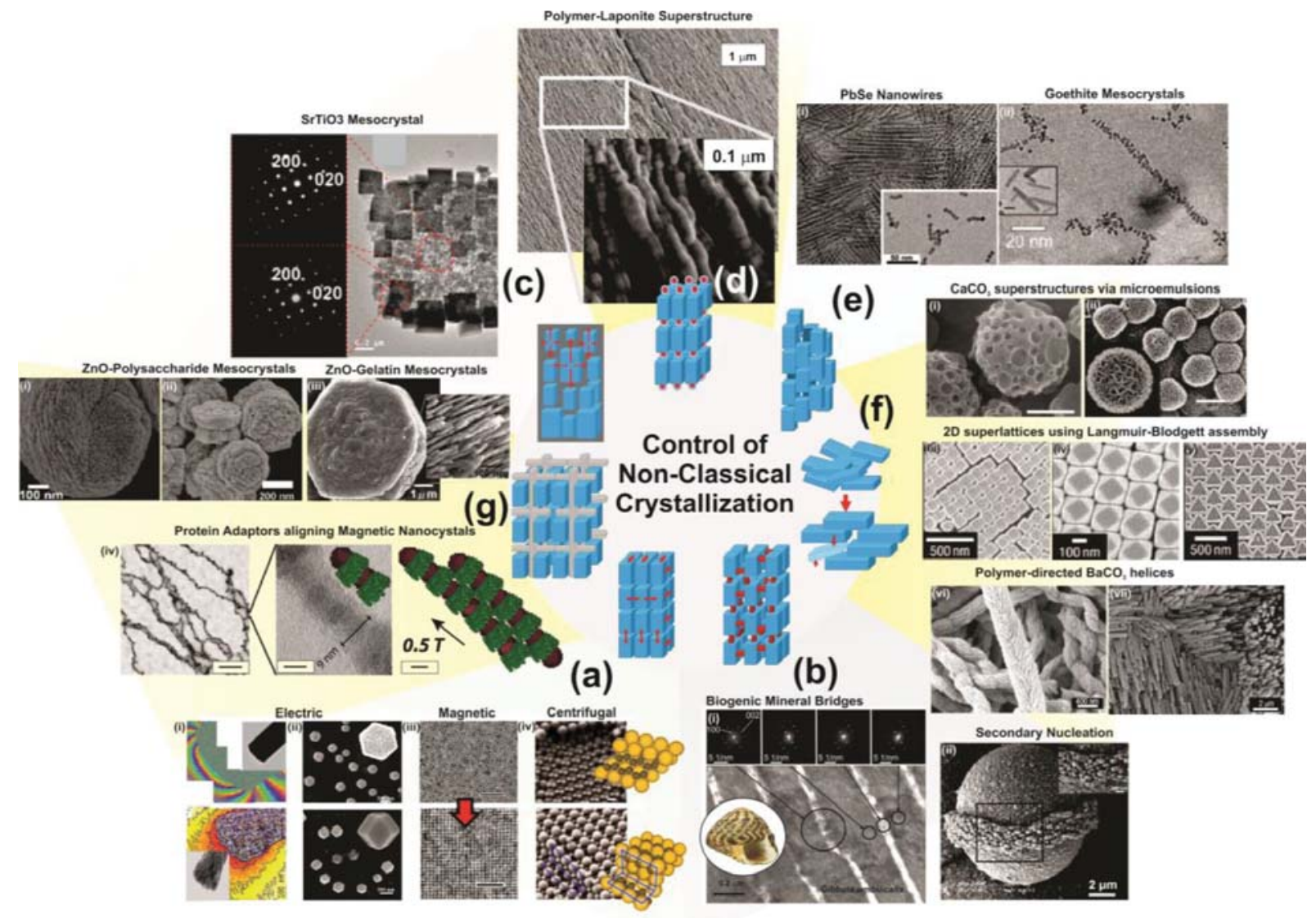

Figure 5. Mechanisms of controlling non-classical crystallization. (a) Alignment by physical forces: (i) intrinsic electric dipole fields of fluorapatite-gelatin nanocomposites, ${ }^{[120]}$ (ii) $\mathrm{Ag}_{2} \mathrm{O}$ mesocrystals of distinct shapes formed in an external electric field, ${ }^{[119]}$ (iii) external magnetic field-controlled ordered superlattice of maghemite nanocubes ${ }^{[121]}$ and (iv) centrifugation-assisted formation of crystal lattices of binary phases. ${ }^{[137]}$ (b) Mineral bridges and secondary nucleation: (i) continuity of crystal lattices of the adjacent tablets comprising a biogenic mineral ${ }^{[126]}$ and (ii) heterostructured microparticles comprising of vaterite cores and equatorial calcite ridges. ${ }^{[129]}$ (c) Topotactic phase transformation: topotactic epitaxy of $\mathrm{SrTiO}_{3}$ mesocrystals originating from $\mathrm{TiO}_{2}$ mesocrystals under hydrothermal conditions. ${ }^{[131]}$ (d) Alignment by particle surface stabilization: rationally designed polymer for directing the long-range organization of laponite particles. ${ }^{[132]}$ (e) Alignment by oriented attachment: (i) PbSe nanowires synthesized by surfactant-controlled attachment of building blocks ${ }^{[57]}$ and (ii) surfactant-devoid goethite mesocrystals. ${ }^{[133]}$ (f) Alignment by spatial constraints: (i) sponge-like vaterite microparticles and (ii) hollow aragonite microspheres formed by using water-in-oil microemulsions, ${ }^{[138]}$ (iii-v) 2D confinement of polyhedral silver nanocrystals into superlattices using the Langmuir-Blodgett method ${ }^{[139]}$ and (vi,vii) helical $\mathrm{BaCO}_{3}$ superstructures formed via the assembly of polymer-stabilized amorphous nanoparticles. ${ }^{[140]}$ (g) Alignment by organic matrix: $\mathrm{ZnO}$ mesocrystals formed in presence of (i) chondroitin sulfate, (ii) hyaluronic acid and (iii) gelatin ${ }^{[141,142]}$ and also (iv) the assembly of $\mathrm{CoFe}_{2} \mathrm{O}_{4}$ nanocrystals in a magnetic field by toroidal protein adaptors. ${ }^{[143]}[$ Reproduced with permissions from (a:i, b:ii, c, f:i, ii) John Wiley and Sons, (a:ii, b:i) Elsevier, (a:iii) National Academy of Sciences, (a:iv, d, e:i, ii, g) American Chemical Society, (f: iii-vii) Springer Nature].

neered for directing particle self-assembly in an inducible and directional manner. For instance, composite layered mesocrystals composed of laponite units are synthesized under influence of a liquid-crystalline polymer (Figure $5 \mathrm{~d}$ ). The polymer structure presents cholesteryl side chains which enables the formation of a chiral nematic liquid crystal phase and also carries carboxyl units that serve as attachment sites for clay platelets. ${ }^{[132]}$ Face-selective nanocrystal stabilizers also regulate oriented attachment mechanisms (Figure 5e) as evident from the attachment of $\mathrm{PbSe}$ nanocrystals to each other on either $\{100\},\{110\}$, or $\{111\}$ faces, under control of surfactant molecules. ${ }^{[57]}$ However crystallographic alignment of nanocrystals can also transiently occur during crystal growth in the absence of surfactants (Figure 5e), on account of solvent occupied inter-particle spaces. ${ }^{[133]}$ The fourth approach i.e. spatial confinement produces increases in local particle densities, thereby promoting inter-particle interactions and driving self-assembly (Figure 5f). This approach is exemplified by reaction environments based on emulsions, solvent evaporation or Langmuir-Blodgett films, which are capable of generating complex organization including $2 \mathrm{D}$ and 3D architectures. ${ }^{[79,134-136]}$ Recent work from our group demonstrates the synthesis of $2 \mathrm{D}$ and $3 \mathrm{D}$ mesocrystalline films via the evaporative self-assembly of iron oxide truncated nanocubes. ${ }^{[79]}$ 
Motivated by the recruitment of amorphous precursors by biogenic crystallization reactions, certain synthetic molecules are rationally designed for controlling phase transformation and crystallization reactions. Double hydrophilic block copolymers (DHBCs) represent a deconstructed version of complex macromolecules regulating biomineralization. ${ }^{[144]}$ These polymers can kinetically stabilize ACC particles and organize mineral particles to achieve hierarchical structures. Determined by the polymer chemistry, particles of atypical shapes and structures are synthesized from amorphous precursors. For example, introduction of phosphonate and sulfonate groups in DHBCs direct the growth of mineral particles towards specific morphologies. ${ }^{[145,146]}$ In some cases, the hierarchical structure of polymeric additives is imprinted upon the emergent hybrid material organization. For instance, the self-assembly of anionic DHBCs and associated material precursors drive the formation of atypical superstructures such as achiral $\mathrm{BaCO}_{3}$ helices ${ }^{[140]}$ (Figure 5f), which can even lead to dynamical Belossov-Zhabotinsky growth patterns, so far only known for solutions. ${ }^{[147]}$ The charged polymer not only confines mineral precursors and controls transformation kinetics, but also defines inter-nanocrystal interactions, imparting hierarchical structuration to the final material similar to biomineralization related proteins and polysaccharides.

Lastly, several biominerals with superior mechanics involve an organic matrix or template, which compartmentalizes and hierarchically organizes the mineral phase. Because of the slower diffusion of ions and ion-clusters in the matrix and also a suppression of convection, the confined volumes within the matrix generally promote nucleation and transformation cascades evident of Ostwald's rule of stages. For instance, the crystallization reactions within the environments of gelatin particles present a clear sequential transformation from an amorphous precursor to an octacalcium phosphate intermediate to hydroxyapatite crystals. ${ }^{[148]}$ The transient stabilization of an amorphous phase is beneficial for its effective transport and infiltration of the nano-spaces within the matrix. At the same time, the confined spaces hinder the attachment of growing nanocrystals and aid the retention of physiochemical properties of nanoparticles in a bulk composite material. ${ }^{[149]}$ A similar process was observed by infiltration of a collagen matrix with calcium phosphate clusters / liquid precursors with some implications onto the understanding of bone growth. ${ }^{[109,150]}$ The matrix structure provides an environment conducive for the formation and prolonged stabilization of superstructures with particulate constituents such as mesocrystals. For these advantages, macromolecules with strong self-association tendencies are suited for the formation of hierarchical material structures. This is evident from the synthesis of biologically-distant zinc oxide mesocrystals by employing biomacromolecules with high self-association tendencies such as hyaluronic acid, chondroitin-6-sulfate and gelatin $^{[141,142]}$ (Figure 5g). An interdependence of the crystallization strategies is indicated by the mineral bridges connecting the lattices of adjacent nanoplates in gelatin-zinc oxide mesocrystals. ${ }^{[141]}$ The structure and assembly of complex macromolecules can also be genetically programmed to achieve the assembly and alignment of nanoparticles in a controlled manner. For instance, recombinant protein adaptors are utilized to align magnetic nanocrystals into nanowires and achieve magnetic coupling between the particles ${ }^{[143]}$ (Figure 5g).

\section{Pathways Towards Functional Materials}

For technological purposes, the production of functional nanostructured materials by means of multistep and energy intensive protocols requiring multiple ingredients can be impractical. However, from thermodynamic and kinetic considerations, a rational engineering of material formation and growth can produce atypical particles and hierarchical architectures with significant yields. As discussed below, these approaches are manifested from the notable works of Prof. Eduardo Ruiz-Hitzky, wherein the nucleation and crystallization of functional hybrid materials are chemically programmed, in several instances by utilizing naturally abundant ingredients. ${ }^{[4,5]}$

\subsection{Molecular Assembly}

The direct production of hierarchical and functional architectures is possible via solute clusters with high propensities towards self-assembly and self-templating. For instance, amphiphilic organosilanes that form micellar assemblies are applied as solute precursors. ${ }^{[151]}$ An optimal hydrolysis of the silica-grafted surfactants produces MCM-41 like mesostructured organizations, directed towards either lamellar or hexagonal long-range periodicity. After thermal treatment, the mesophases yield distinct silica mesoporous organizations. In relation, reactions involving mixtures of silanes and surfactants might produce relatively less complex structures such as hollow micro-particles. ${ }^{[152]}$ Thus, an appropriate choice of soluble precursors can guide the organization of the mesostructured materials across large length scales.

In the organic matrix of certain biominerals, ${ }^{[153,154]}$ surface-immobilized biomacromolecules can locally enrich solute precursors and serve as biochemically-encoded nucleation sites. Analogous to this biological strategy, functional hierarchical materials are achieved by the heterogeneous nucleation of particles on surfaces with defined physicochemical properties. With this approach, titanosilicate-glass fiber composites are synthesized via hydrothermal reactions under alkaline conditions (Figure 6a). At high $\mathrm{pH}$ conditions, the 

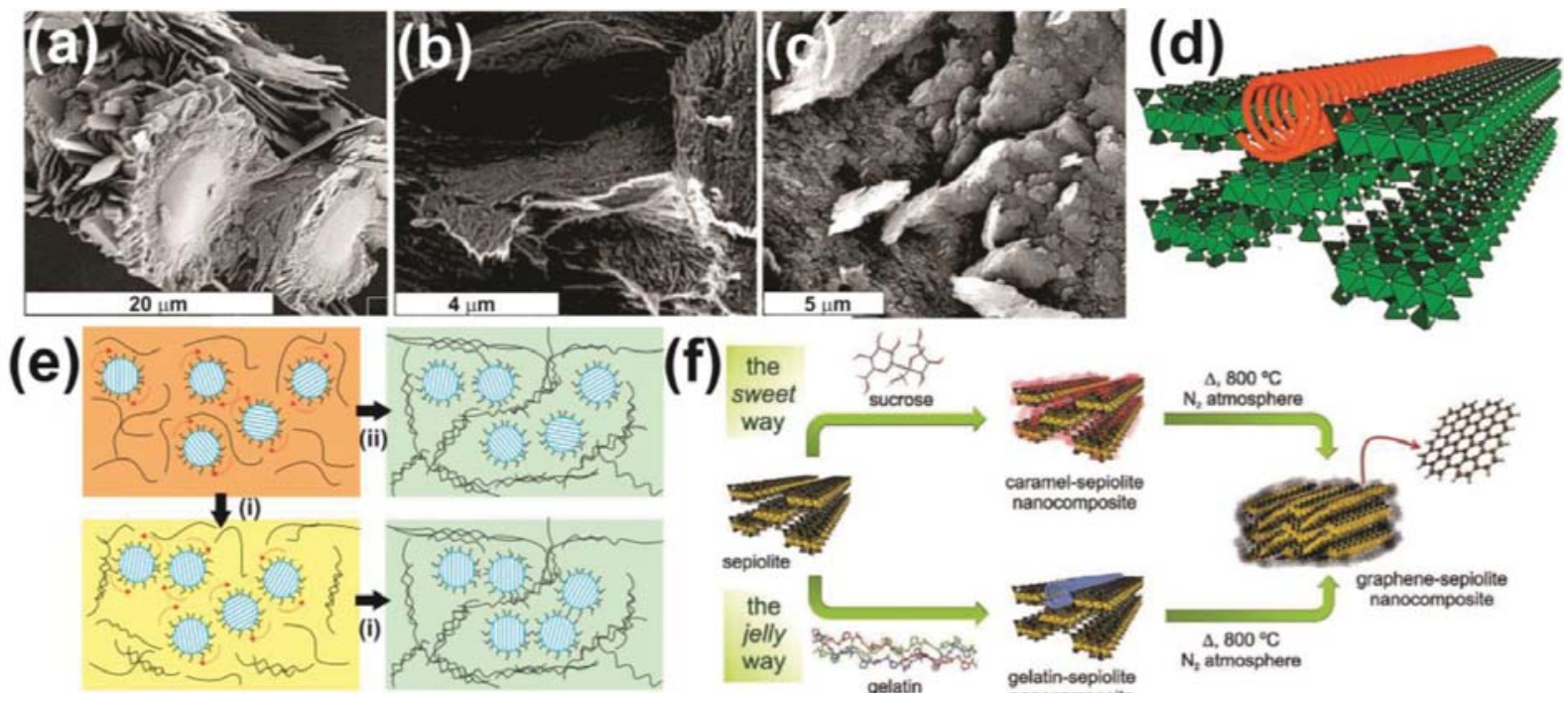

Figure 6. (a) Functional heterostructures formed by the radial growth of titanosilicate crystals on glass fibres, ${ }^{[155]}$ (b) layered organization of chitosan-sepiolite nanostructured films synthesized by evaporative assembly, ${ }^{[158]}$ (c) compact arrangement of carrageenan- $\left[\mathrm{Zn}_{2} \mathrm{Al}\right]$ nanocomposites ${ }^{[159]}$ and $(\mathrm{d})$ representation of the interfacial complementarity between sepiolite tunnels and gelatin triple helix domains. ${ }^{[160]}$ (e) Cartoon depicting the mobility and co-orientation (red arrows) of nanoparticles confined in gel networks, ${ }^{[161]}$ wherein (i) a slow gelation produces open network structures at the nanoscale and do not relatively affect inter-particle interactions and mobility whereas (ii) rapid gel formation enhances the rotational hydrodynamic resistance of the nanocrystals supressing the co-alignment of particles. (f) Synthetic scheme for graphene preparation based on either the infiltration of sucrose-derived caramel in the sepiolite structure or the assembly of gelatin-sepiolite nanocomposites. ${ }^{[162]}$ Subsequent thermal treatment in $\mathrm{N}_{2}$ atmospheres produces hybrid structures with graphene layers (N-doped when gelatinderived) supported on clay materials. [Reproduced with permissions from (a,c) American Chemical Society, (b) Elsevier, (d, e) Royal Society of Chemistry, (f) John Wiley and Sons].

lixiviation of silica from the glass fiber templates promotes the cementation of a growing titanosilicate phase with the glass substrate, generating stable hetero-structures with hydrogen adsorption and photo-catalytic capacities. ${ }^{[155,156]}$ In another instance, the surfaces of microfibrous sepiolite are treated with a cationic surfactant to achieve the heterogeneous nucleation of $\mathrm{TiO}_{2}$ nanoparticles. ${ }^{[157]}$ During situ hydrolysis, the organophilic surfaces of modified sepiolite spatially regulate the nucleation of $\mathrm{TiO}_{2}$ particles from alkoxides precursors. Thus, by an appropriate choice of solute precursor and reaction interfaces, the mechanisms of heterogeneous nucleation towards nanostructured composites can be effectively controlled.

\subsection{Evaporative Particle Assembly}

Abundant in the Earth's crust and with distinct structures and surface chemistry, clay minerals have potential contributions in chemical transformations of primordial significance involving adsorbed solute and organic molecules. ${ }^{[163]}$ The compositional and structural diversity of clay materials also produce distinct physicochemical properties and reactivity. For instance, although kaolins, smectites and palygorskite present octahedral and tetrahedral sheets as basic building constituents, their structure and composition as well as related properties of adsorption, color, isoelectric point, surface area and viscosity are quite dissimilar. ${ }^{[164]}$ Based on this outlook, clay minerals are promising materials for fabricating diverse functional nanocomposites. ${ }^{165]}$

Clay-polymer nanostructured composites are mechanically robust materials with interesting physicochemical properties suited for applications in catalysis, environmental remediation as well as therapeutic and biomedical devices. ${ }^{[4,165-167]}$ Applied as inorganic constituents, clay minerals offer several benefits including (i) well-characterized compositions, morphologies and crystallographic properties, (ii) excellent ion-exchange capacities and high specific surface area as well as (iii) high chemical resistance and thermal stabilities. ${ }^{[166]}$ On the other hand, due to their biocompatibility and natural abundance, biopolymers are interesting alternates to synthetic counterparts applied for the controlled assembly of particles and mechanical reinforcement of the composites. For these reasons, fibrous clay minerals are assembled by using polysaccharides with distinct charge properties viz. alginate, chitosan and starch. ${ }^{[158]}$ Following a simple fabrication protocol, the clay-polysaccharide composites are derived by an initial adsorption of the polysaccharides on the surfaces of mineral particles, followed by an evaporation-induced assembly. With polysaccharide-determined morphological patterns, chitosan and starch strongly associate with the mineral particles and exhibit compact arrangements (Figure 6b). This is attributed to hydrogen bonding interactions between the 
macromolecules and the silanol groups of the mineral phase. The resultant nanostructured hybrids not only provide enhanced water resistance and mechanics, but also enable the polysaccharide filler to retain potent metal adsorption capacities. ${ }^{[158]}$ The practicality of this two-step fabrication approach (i.e. surface modification and evaporation-driven assembly) towards functional materials is also illustrated by chitosan-montmorillonite nanocomposites applied in electrochemical sensors and by sacran-sepiolite hybrids exhibiting promising biosorbent behavior. ${ }^{[168,169]}$ For organic fillers with intrinsically high hydration contents, freeze-drying procedures can accelerate the co-assembly of particles and organic fillers. ${ }^{[170]}$ In all, evaporative self-assembly emerges as a rapid, straightforward and versatile methodology to transform micro- and nano-particles into functional composites. The process of evaporation not only constraints the primary particles to smaller volumes with increasing time but also promotes the polymer-controlled directional assembly of building units, producing organized nanostructures distributed across macroscopic length scales. ${ }^{[74,79,171]}$

The integration of heterogeneous constituents into nanostructured composites is also achieved by combining the nucleation and assembly of particles under the influence of macromolecules. Based on their chemical functionality, molar mass and molecular organization, macromolecules serve multiple functions including (i) the confinement of the reactants directing emergent particle shapes, sizes, polymorphs and textures, ${ }^{[172,173]}$ (ii) the stabilization of high energy surfaces of nanocrystals ${ }^{[174]}$ and (iii) the introduction of long range order to particle assemblies. ${ }^{[132]}$ This approach is exemplified by the synthesis of layered double hydroxides (LDHs) intercalated with polysaccharides ${ }^{[159]}$ (Figure 6c). The $\mathrm{LDH}$ particles are initially precipitated from solute precursors, during which the anionic biopolymers are effectively intercalated in the layered structure via electrostatic interactions. Subsequent assembly of the biopolymer-stabilized sheets is done by centrifugation and evaporative assembly. In the resultant hybrid material, an effective integration of the $\mathrm{LDH}$ and polysaccharide constituents is reflected by the reversal of the anion exchange behavior typical of unmodified LDH. Interestingly, among the tested polysaccharides, alginate and pectin form monolayers whereas t-carrageenan is incorporated as a bilayer or a double helix in the composite structure. Besides, the interactions and intercalation of polymers also affect the crystallinity, shape and size of the LDH host material. ${ }^{[175]}$ Thus, the interfacial functional and structural complementarity between the organic and inorganic constituents of hybrid materials emerges as a crucial factor in determining the molecular and structural integration of the constitutive phases as well as the mechanics of the composite material.

\subsection{Particle Assembly via Thermal Transitions of Polymers}

Certain macromolecules exhibit thermal transitions of conformation and self-association, which can be tactically applied for directing particle nucleation, growth and assembly. On account of its biocompatibility, cost benefit, ease of processability and aqueous solubility, gelatin is widely explored as a thermo-reversible gelling material for fabricating diverse hybrid materials. Another advantage is the dependency of the transition temperature of gelatin on conditions of thermal history, $\mathrm{pH}$ as well as the presence of soluble and insoluble constituents. ${ }^{[176]}$ For instance, distinct variations in the thermal transitions of gelatin are observed in mixtures containing clay materials such as sepiolite, laponite and cloisite, representing an important process parameter in fabricating nanostructured composites. ${ }^{[177]}$ With the structural composition of the gelatin molecules affecting emergent mechanics, ${ }^{[178]}$ further investigations reflect the potential of engineering organic-inorganic interfaces for fabricating robust hybrid materials. For example, systematic variations in the structure and surface of mineral fillers induce distinct degrees of renaturation of the gelatin matrix. ${ }^{[160]}$ Specifically, in relation to chemically and thermally modified sepiolite variants, the untreated clay minerals not only enhance the renaturation of the gelatin matrix but also produce nanocomposites with better elastic properties. The reinforcement of the organic-inorganic interfaces and the specific renaturation of macromolecules are suggested to originate from molecular alignment and structural complementarity between the triple helix of gelatin and sepiolite surfaces ${ }^{[160]}$ (Figure 6d). The mineral surfaces shift the equilibrium towards gelatin's crystalline structure in course of the thermal transition, which might elucidate the $\mathrm{pH}$ and temperature dependent trends of gelatin adsorption to raw clay particles. ${ }^{[179]}$

The non-classical crystallization of functional composites based on the sol-gel transition of gelatin is demonstrated by perovskite-based hybrid materials. ${ }^{[180]}$ The synthesis procedure involves an initial delamination of the perovskite material into colloidal nanosheets stabilized by alklyammonium molecules. Particle assembly is then achieved by a two-step protocol involving (i) the melting and dispersion of gelatin in the presence of inorganic nanosheets to obtain homogenous mixtures and (ii) incubating the mixtures at room temperature to achieve a slow evaporation of water and a gradual onset of the sol-gel transition. Occurring within the gelatin matrix, the assembly of nanosheets leads to a crystallographically-oriented, stacking arrangement of perovskite nanosheets, indicative of mesocrystalline architectures. ${ }^{[180]}$ The gelatin-perovskite composites also exhibit dielectric constants tunable by the relative organic and inorganic compositions, which along with the moldabililty of the 
precursor mixtures might be suited for the development of electrochemical films and coatings. Since the gelation mechanism involves distinct contributions from macroscopic and nanoscopic rheological changes, a gradual drop in temperature enables the formation of flexible local networks that enable the rotation and possibly also the co-orientation of confined nanoparticles ${ }^{[161]}$ (Figure 6e). Additionally, the decreasing temperature generates a certain driving force for re-crystallization, which when rapidly performed can inhibit the mutual orientation of the particulate units. ${ }^{[102]}$ Thus, slow rates of cooling can produce larger domains of crystallographically aligned nanoparticles, which are highly desired for the fabrication of functional nanostructured composites.

\subsection{Clay Minerals for Graphene Assembly}

Graphene, the 2D allotrope of carbon, exhibits spectacular electronic and mechanical properties originating from a structure of carbon atoms limited to a single plane. ${ }^{[181,182]}$ Due its commercial importance, a need exists for the largescale synthesis of graphene via economic and scalable methods. Methods based on mechanical exfoliation and aggressive solvents are usually limited to the production and handling of small amounts of graphene. A solution is provided by the template-directed synthesis of graphene-like materials from naturally abundant precursors in the confined volume and defined interfaces presented by layered solids (Figure 6f). For instance, by using microwave irradiation, the intercalation of sucrose-derived caramel in montmorillonite produces composites, which on carbonization in a nonoxidizing atmosphere produce nanocomposites constituted with graphene-like materials. ${ }^{[162,183]}$ From X-ray diffraction, the interlayer distance of the composites is estimated at about $0.4 \mathrm{~nm}$, indicative of graphene monolayers associated with the layered silicate. Significant increases in conductivity from $10^{-12}$ to $10^{-1} \mathrm{~S} \mathrm{~cm}^{-1}$ after thermal treatment are suggestive of uniformly distributed graphene sheets in the template volume. ${ }^{[183]}$ Thus, the fluidic properties of caramel appear to aid an effective infiltration of the clay structure. In view of the silicate template, the silanol groups can be utilized for the introduction of reactive groups and functionality in the composite material, for example by using organosilanes. ${ }^{[184]}$ Another advantage of the clay-based template is the possibility to tune its surface properties in order to achieve distinct microstructures and mechanics of the emergent 2D materials. ${ }^{[185]}$ This synthesis procedure can also be applied for the production of nitrogen doped graphene, wherein the saccharide precursor is substituted with gelatin. ${ }^{[162]}$

\section{Conclusion}

Synthetic challenges in material science range from controlling the dynamics, size and shape of nanoscopic forms to regulating the interfaces and organizations of matter across hierarchical levels. Recent progresses in the field of nucleation and crystallization identify a multiplicity of ingredients in form of solutes, fluids, gels and particles, which not only present unique interactions and interfaces but also exhibit distinct susceptibilities to physicochemical manipulation. Avenues for the strategic control of structural organization at distinct length scales are provided by these multistep and multicomponent pathways of material nucleation and growth. Illustrated by diverse materials, in this account, the extent of morphological, compositional and structural complexity accessible by regulating immediate and distant precursors is remarkable. Since an enormous but underexplored potential exists for guiding the form, structure and stability of material precursors and intermediates towards complex material organizations, there is as yet plenty of room "at the bottom" as well as "between the bottom and top" ${ }^{[186]}$ Keeping in mind that knowledge transfer from basic concepts to technological solutions is a cornerstone of materials science, as reflected by the works of Prof. Eduardo Ruiz-Hitzky, the pathways of nucleation and crystallization can be effectively guided towards functional nanostructured materials with ecological and economic considerations.

\section{Acknowledgements}

A.R. acknowledges a fellowship from the Freiburg Institute for Advanced Studies.

\section{References}

[1] J. J. De Yoreo, P. U. Gilbert, N. A. Sommerdijk, R. L. Penn, S. Whitelam, D. Joester, H. Zhang, J. D. Rimer, A. Navrotsky, J. F. Banfield, Science 2015, 349, aaa6760.

[2] F. C. Meldrum, H. Cölfen, Chem. Rev. 2008, 108, 43324432.

[3] C. Rodriguez-Navarro, A. Burgos Cara, K. Elert, C. V. Putnis, E. Ruiz-Agudo, Cryst. Growth Des. 2016, 16, 1850 1860.

[4] E. Ruiz-Hitzky, M. Darder, P. Aranda, K. Ariga, Adv. Mater. 2010, 22, 323-336.

[5] M. Darder, P. Aranda, E. Ruiz-Hitzky, Adv. Mater. 2007, 19, 1309-1319.

[6] Y. Politi, T. Arad, E. Klein, S. Weiner, L. Addadi, Science 2004, 306, 1161-1164.

[7] D. Gebauer, A. Völkel, H. Cölfen, Science 2008, 322, 18191822.

[8] F. C. Meldrum, R. P. Sear, Science 2008, 322, 1802-1803. 
[9] F. Sebastiani, S. L. Wolf, B. Born, T. Q. Luong, H. Cölfen, D. Gebauer, M. Havenith, Angew. Chem. Int. Ed. 2017, 56, 490-495.

[10] A. Schiener, A. Magerl, A. Krach, S. Seifert, H.-G. Steinrück, J. Zagorac, D. Zahn, R. Weihrich, Nanoscale 2015, 7, $11328-11333$.

[11] C. M. Völkle, D. Gebauer, H. Cölfen, Faraday Discuss. 2015, 179, 59-77.

[12] W. W. Laxson, R. G. Finke, J. Am. Chem. Soc. 2014, 136, 17601-17615.

[13] G. Zhang, C. Liu, D.-L. Long, L. Cronin, C.-H. Tung, Y. Wang, J. Am. Chem. Soc. 2016, 138, 11097-11100.

[14] J. Scheck, B. Wu, M. Drechsler, R. Rosenberg, A. E. Van Driessche, T. M. Stawski, D. Gebauer, J. Phys. Chem. Lett. 2016, 7, 3123-3130.

[15] J. Lee, J. Yang, S. G. Kwon, T. Hyeon, Nat. Rev. Mater. 2016, 1,16034 .

[16] G. Schmid, Chem. Rev. 1992, 92, 1709-1727.

[17] M. R. Friedfeld, J. L. Stein, B. M. Cossairt, Inorg. Chem. 2017.

[18] D. J. Smith, A. K. Petford-Long, L. Wallenberg, J. Bovin, Science 1986, 233, 872-875.

[19] Y. Lu, W. Chen, Chem. Soc. Rev. 2012, 41, 3594-3623.

[20] Y. Yang, H. Zhang, J. F. Douglas, ACS Nano 2014, 8, 74657477.

[21] J. Liu, N. Jian, I. Ornelas, A. J. Pattison, T. Lahtinen, K. Salorinne, H. Häkkinen, R. E. Palmer, Ultramicroscopy 2017, $176,146-150$.

[22] J. H. Yu, X. Liu, K. E. Kweon, J. Joo, J. Park, K.-T. Ko, D. W. Lee, S. Shen, K. Tivakornsasithorn, J. S. Son, Nat. Mater. 2010, 9, 47-53.

[23] D. Gebauer, M. Kellermeier, J. D. Gale, L. Bergström, H. Cölfen, Chem. Soc. Rev. 2014, 43, 2348-2371.

[24] D. Gebauer, P. N. Gunawidjaja, J. Ko, Z. Bacsik, B. Aziz, L. Liu, Y. Hu, L. Bergström, C. W. Tai, T. K. Sham, Angew. Chem. 2010, 122, 9073-9075.

[25] S. Sun, D. M. Chevrier, P. Zhang, D. Gebauer, H. Cölfen, Angew. Chem. Int. Ed. 2016, 55, 12206-12209.

[26] S. Sun, D. Gebauer, H. Cölfen, Chem. Sci. 2017, 8, 14001405.

[27] R. Demichelis, P. Raiteri, J. D. Gale, D. Quigley, D. Gebauer, Nat. Commun. 2011, 2, 590.

[28] M. Kellermeier, P. Raiteri, J. K. Berg, A. Kempter, J. D. Gale, D. Gebauer, ChemPhysChem 2016, 17, 3535-3541.

[29] A. Burgos-Cara, C. V. Putnis, C. Rodriguez-Navarro, E. Ruiz-Agudo, Minerals 2017, 7, 126.

[30] A. Rao, D. Gebauer, H. Cölfen, Crystals 2017, 7, 0302.

[31] M. Walther, D. Zahn, Eur. J. Inorg. Chem. 2015, 2015, 1178-1181.

[32] N. D. Loh, S. Sen, M. Bosman, S. F. Tan, J. Zhong, C. A. Nijhuis, P. Král, P. Matsudaira, U. Mirsaidov, Nat. Chem. 2016.

[33] S. J. Homeijer, R. A. Barrett, L. B. Gower, Cryst. Growth Des. 2010, 10, 1040-1052.

[34] Y. Jiang, L. Gower, D. Volkmer, H. Cölfen, Phys. Chem. Chem. Phys. 2012, 14, 914-919.

[35] A. Rao, H. Cölfen, Biophysical reviews 2016, 1-21.
[36] A. Saito, K. Igarashi, M. Azuma, H. Ooshima, J. Chem. Eng. Jpn. 2002, 35, 1133-1139.

[37] W. Tang, M. Zhang, H. Mo, J. Gong, J. Wang, T. Li, Cryst. Growth Des. 2017, 17, 5049-5053.

[38] A. Soltani, D. Gebauer, L. Duschek, B. Fischer, H. Cölfen, M. Koch, Chemistry 2017.

[39] M. Kellermeier, R. Rosenberg, A. Moise, U. Anders, M. Przybylski, H. Cölfen, Faraday Discuss. 2012, 159, 23-45.

[40] L. B. Gower, D. J. Odom, J. Cryst. Growth 2000, 210, 719734.

[41] Y. Jiang, L. Gower, D. Volkmer, H. Cölfen, Cryst. Growth Des. 2011, 11, 3243-3249.

[42] S. L. Wolf, L. Caballero, F. Melo, H. Cölfen, Langmuir 2016 , 33, 158-163.

[43] L. B. Gower, Chem. Rev. 2008, 108, 4551-4627.

[44] M. A. Bewernitz, D. Gebauer, J. Long, H. Cölfen, L. B. Gower, Faraday Discuss. 2012, 159, 291-312.

[45] S. E. Wolf, L. B. Gower, in New Perspectives on Mineral Nucleation and Growth, Springer, 2017, pp. 43-75.

[46] A. F. Wallace, L. O. Hedges, A. Fernandez-Martinez, P. Raiteri, J. D. Gale, G. A. Waychunas, S. Whitelam, J. F. Banfield, J. J. De Yoreo, Science 2013, 341, 885-889.

[47] J. Rieger, T. Frechen, G. Cox, W. Heckmann, C. Schmidt, J. Thieme, Faraday Discuss. 2007, 136, 265-277.

[48] M. Faatz, F. Gröhn, G. Wegner, Adv. Mater. 2004, 16, 9961000.

[49] J. W. Cahn, Acta Metall. 1961, 9, 795-801.

[50] S. E. Wolf, L. Müller, R. Barrea, C. J. Kampf, J. Leiterer, U. Panne, T. Hoffmann, F. Emmerling, W. Tremel, Nanoscale 2011, 3, 1158-1165.

[51] S. S. Jee, R. K. Kasinath, E. DiMasi, Y.-Y. Kim, L. Gower, CrystEngComm 2011, 13, 2077-2083.

[52] B. Wingender, P. Bradley, N. Saxena, J. W. Ruberti, L. Gower, Matrix Biol. 2016, 52, 384-396.

[53] Y. Ma, G. Mehltretter, C. Plüg, N. Rademacher, M. U. Schmidt, H. Cölfen, Adv. Funct. Mater. 2009, 19, 20952101.

[54] H. Cöelfen, M. Antonietti, Mesocrystals and nonclassical crystallization. Editor, John Wiley \& Sons, 2008.

[55] M. Niederberger, H. Cölfen, Phys. Chem. Chem. Phys. 2006, 8, 3271-3287.

[56] S. E. Wolf, C. F. Böhm, J. Harris, B. Demmert, D. E. Jacob, M. Mondeshki, E. Ruiz-Agudo, C. Rodríguez-Navarro, J. Struct. Biol. 2016, 196, 244-259.

[57] K.-S. Cho, D. V. Talapin, W. Gaschler, C. B. Murray, J. Am. Chem. Soc. 2005, 127, 7140-7147.

[58] R. L. Penn, J. F. Banfield, Science 1998, 281, 969-971.

[59] D. Li, M. H. Nielsen, J. R. Lee, C. Frandsen, J. F. Banfield, J. J. De Yoreo, Science 2012, 336, 1014-1018.

[60] N. Gehrke, H. Cölfen, N. Pinna, M. Antonietti, N. Nassif, Cryst. Growth Des. 2005, 5, 1317-1319.

[61] W. T. Read, W. Shockley, Phys. Rev. 1950, 78, 275.

[62] C. G. Dunn, F. Lionetti, JOM 1949, 1, 125-132.

[63] H. Zhang, J. F. Banfield, CrystEngComm 2014, 16, 15681578.

[64] D. V. Talapin, E. V. Shevchenko, C. B. Murray, A. V. Titov, P. Král, Nano Lett. 2007, 7, 1213-1219. 
[65] Z. Tang, N. A. Kotov, M. Giersig, Science 2002, 297, 237240.

[66] W. Lv, W. Huo, Y. Niu, Y. Zhu, Y. Xie, X. Guo, W. He, CrystEngComm 2015, 17, 729-733.

[67] H. Cölfen, M. Antonietti, Angew. Chem. Int. Ed. 2005, 44, 5576-5591.

[68] M.-G. Ma, H. Cölfen, Current Opinion in Colloid \& Interface Science 2014, 19, 56-65.

[69] J. Fang, B. Ding, H. Gleiter, Chem. Soc. Rev. 2011, 40, $5347-5360$

[70] L. Bahrig, S. G. Hickey, A. Eychmüller, CrystEngComm 2014, 16, 9408-9424.

[71] T. Tachikawa, T. Majima, NPG Asia Mater. 2014, 6, e100.

[72] L. Bergström, E. V. Sturm, G. Salazar-Alvarez, H. Cölfen, Acc. Chem. Res. h 2015, 48, 1391-1402.

[73] E. Uchaker, G. Cao, Nano Today 2014, 9, 499-524.

[74] M. Agthe, T. S. Plivelic, A. Labrador, L. Bergström, G. Salazar-Alvarez, Nano Lett. 2016, 16, 6838-6843.

[75] J. Seto, Y. Ma, S. A. Davis, F. Meldrum, A. Gourrier, Y.-Y. Kim, U. Schilde, M. Sztucki, M. Burghammer, S. Maltsev, Proc. Natl. Acad. Sci. USA 2012, 109, 3699-3704.

[76] Y. Politi, R. A. Metzler, M. Abrecht, B. Gilbert, F. H. Wilt, I. Sagi, L. Addadi, S. Weiner, P. Gilbert, Proc. Natl. Acad. Sci. USA 2008, 105, 17362-17366.

[77] A. Rao, H. Cölfen, in New Perspectives on Mineral Nucleation and Growth, Springer, 2017, pp. 155-183.

[78] D. Schwahn, Y. Ma, H. Cölfen, J. Phys. Chem. C 2007, 111, 3224-3227.

[79] J. Brunner, I. A. Baburin, S. Sturm, K. Kvashnina, A. Rossberg, T. Pietsch, S. Andreev, H. Cölfen, Adv. Mater. Interfaces 2017, 4.

[80] L. Addadi, S. Raz, S. Weiner, Adv. Mater. 2003, 15, 959970.

[81] J. H. Cartwright, A. G. Checa, J. D. Gale, D. Gebauer, C. I. Sainz-Díaz, Angew. Chem. Int. Ed. 2012, 51, 11960-11970.

[82] G. Wulff, Ger. Z. Kristallogr 1901, 34, 449-530.

[83] A. Putnis, Mineral. Mag. 2002, 66, 689-708.

[84] A. Putnis, C. V. Putnis, J. Solid State Chem. 2007, 180, $1783-1786$.

[85] A. Gal, K. Kahil, N. Vidavsky, R. T. De Vol, P. U. Gilbert, P. Fratzl, S. Weiner, L. Addadi, Adv. Funct. Mater. 2014, 24, 5420-5426.

[86] S. Sun, D. Gebauer, H. Cölfen, Angew. Chem. Int. Ed. 2017, 56, 4042-4046.

[87] B. Guillemet, M. Faatz, F. Gröhn, G. Wegner, Y. Gnanou, Langmuir 2006, 22, 1875-1879.

[88] A. Radha, A. Fernandez-Martinez, Y. Hu, Y.-S. Jun, G. A. Waychunas, A. Navrotsky, Geochim. Cosmochim. Acta 2012, 90, 83-95.

[89] H. Cölfen, Curr. Opin. Colloid Interface Sci. 2003, 8, 23-31.

[90] C. Li, L. Qi, Angew. Chem. Int. Ed. 2008, 47, 2388-2393.

[91] A. Navrotsky, Proc. Natl. Acad. Sci. U.S.A. 2004, 101, 12096-12101.

[92] H. Häkkinen, M. Walter, H. Grönbeck, J. Phys. Chem. B 2006, 110, 9927-9931.

[93] M. G. Taylor, G. Mpourmpakis, Nat. Commun. 2017, 8, 15988.
[94] N. Toshima, Pure Appl. Chem. 2000, 72, 317-325.

[95] Y. Yu, Z. Luo, D. M. Chevrier, D. T. Leong, P. Zhang, D.-E. Jiang, J. Xie, J. Am. Chem. Soc. 2014, 136, 1246-1249.

[96] G. Li, H. Abroshan, C. Liu, S. Zhuo, Z. Li, Y. Xie, H. J. Kim, N. L. Rosi, R. Jin, ACS Nano 2016, 10, 7998-8005.

[97] E. Ruiz-Agudo, A. Burgos-Cara, C. Ruiz-Agudo, A. IbañezVelasco, H. Cölfen, C. Rodriguez-Navarro, Nat. Commun. 2017, 8, 768 .

[98] A. Rao, Y.-C. Huang, H. Cölfen, J. Phys. Chem. C 2017, 121, 21641-21649.

[99] A. Verch, M. Antonietti, H. Cölfen, Z. Kristallogr. Cryst. Mater. 2012, 227, 718-722.

[100] D. C. Gary, M. W. Terban, S. J. Billinge, B. M. Cossairt, Chem. Mater. 2015, 27, 1432-1441.

[101] S. Tamang, S. Lee, H. Choi, S. Jeong, Chem. Mater. 2016, $28,8119-8122$.

[102] Y. Jiang, H. Gong, M. Grzywa, D. Volkmer, L. Gower, H. Cölfen, Adv. Funct. Mater. 2013, 23, 1547-1555.

[103] Y.-Y. Kim, E. P. Douglas, L. B. Gower, Langmuir 2007, 23, $4862-4870$.

[104] X. Cheng, L. B. Gower, Biotechnol. Prog. 2006, 22, 141-149.

[105] S. Wohlrab, H. Cölfen, M. Antonietti, Angew. Chem. Int. Ed. 2005, 44, 4087-4092.

[106] Y. Jiang, M. Kellermeier, D. Gebauer, Z. Lu, R. Rosenberg, A. Moise, M. Przybylski, H. Cölfen, Nat. Commun. 2017, 8, ncomms 15933 .

[107] M. J. Olszta, D. J. Odom, E. P. Douglas, L. B. Gower, Connect. Tissue Res. 2003, 44, 326-334.

[108] S. J. Homeijer, M. J. Olszta, R. A. Barrett, L. B. Gower, J. Cryst. Growth 2008, 310, 2938-2945.

[109] F. Nudelman, K. Pieterse, A. George, P. H. Bomans, H. Friedrich, L. J. Brylka, P. A. Hilbers, N. A. Sommerdijk, Nat. Mater. 2010, 9, 1004-1009.

[110] T. Wang, A. Verch, H. G. Boerner, H. Coelfen, M. Antonietti, J. Ceram. Soc. Jpn. 2009, 117, 221-227.

[111] L. Dai, E. P. Douglas, L. B. Gower, J. Non-Cryst. Solids 2008, 354, 1845-1854.

[112] L. Dai, X. Cheng, L. B. Gower, Chem. Mater. 2008, 20, 6917-6928.

[113] A. Rao, Mater. Today 2017, 20, 220-221 https://doi.org/ 10.1016/j.mattod.2017.03.020.

[114] U. Gasser, J. Phys. Condens. Matter 2009, 21, 203101.

[115] P. Tan, N. Xu, L. Xu, Nat. Phys. 2014, 10, 73-79.

[116] Y. Min, M. Akbulut, K. Kristiansen, Y. Golan, J. Israelachvili, Nat. Mater. 2008, 7, 527-538.

[117] E. V. Sturm, H. Cölfen, Chem. Soc. Rev. 2016, 45, 58215833.

[118] R. Q. Song, H. Cölfen, Adv. Mater. 2010, 22, 1301-1330.

[119] J. Fang, P. M. Leufke, R. Kruk, D. Wang, T. Scherer, H. Hahn, Nano Today 2010, 5, 175-182.

[120] P. Simon, D. Zahn, H. Lichte, R. Kniep, Angew. Chem. 2006, 118, 1945-1949.

[121] A. Ahniyaz, Y. Sakamoto, L. Bergström, Proc. Natl. Acad. Sci. USA 2007, 104, 17570-17574.

[122] E. Wetterskog, A. Klapper, S. Disch, E. Josten, R. P. Hermann, U. Rücker, T. Brückel, L. Bergström, G. SalazarAlvarez, Nanoscale 2016, 8, 15571-15580. 
[123] R. Song, T. Krasia-Christoforou, C. Debus, H. Cölfen, Small 2017, 13.

[124] C. Lausser, H. Colfen, M. Antonietti, ACS Nano 2011, 5, 107-114.

[125] H.-L. Gao, S.-M. Chen, L.-B. Mao, Z.-Q. Song, H.-B. Yao, H. Cölfen, X.-S. Luo, F. Zhang, Z. Pan, Y.-F. Meng, Nat. Commun. 2017, 8, 287.

[126] A. G. Checa, J. H. Cartwright, M.-G. Willinger, J. Struct. Biol. 2011, 176, 330-339.

[127] Y. Oaki, A. Kotachi, T. Miura, H. Imai, Adv. Funct. Mater. 2006, 16, 1633-1639.

[128] N. Nassif, N. Pinna, N. Gehrke, M. Antonietti, C. Jäger, H. Cölfen, Proc. Natl. Acad. Sci. USA 2005, 102, 12653-12655.

[129] S. S. Wang, A. Picker, H. Cölfen, A. W. Xu, Angew. Chem. Int. Ed. 2013, 52, 6317-6321.

[130] V. Kalyani, B. S. Vasile, A. Ianculescu, M. T. Buscaglia, V. Buscaglia, P. Nanni, Cryst. Growth Des. 2012, 12, 44504456.

[131] P. Zhang, T. Ochi, M. Fujitsuka, Y. Kobori, T. Majima, T. Tachikawa, Angew. Chem. Int. Ed. 2017, 56, 5299-5303.

[132] U. Tritschler, I. Zlotnikov, P. Zaslansky, B. Aichmayer, P. Fratzl, H. Schlaad, H. Cölfen, Langmuir 2013, 29, 1109311101.

[133] V. M. Yuwono, N. D. Burrows, J. A. Soltis, R. L. Penn, J. Am. Chem. Soc. 2010, 132, 2163-2165.

[134] A. R. Tao, J. Huang, P. Yang, Acc. Chem. Res. 2008, 41, 1662-1673.

[135] M. Li, S. Mann, Adv. Funct. Mater. 2002, 12, 773-779.

[136] M. Agthe, E. Wetterskog, J. Mouzon, G. Salazar-Alvarez, L. Bergström, CrystEngComm 2014, 16, 1443-1450.

[137] M. Chen, H. Cölfen, S. Polarz, ACS Nano 2015, 9, 69446950.

[138] S. Mann, Angew. Chem. Int. Ed. 2000, 39, 3392-3406.

[139] A. Tao, P. Sinsermsuksakul, P. Yang, Nat. Nanotechnol. 2007, 2, 435-440.

[140] S.-H. Yu, H. Cölfen, K. Tauer, M. Antonietti, Nat. Mater. 2005, 4, 51-55.

[141] Y.-H. Tseng, H.-Y. Lin, M.-H. Liu, Y.-F. Chen, C.-Y. Mou, J. Phys. Chem. C 2009, 113, 18053-18061.

[142] F. Waltz, G. Wißmann, J. Lippke, A. M. Schneider, H.-C. Schwarz, A. Feldhoff, S. Eiden, P. Behrens, Cryst. Growth Des. 2012, 12, 3066-3075.

[143] T. A. Pham, A. Schreiber, S. M. Schiller, H. Cölfen, $A d v$. Funct. Mater. 2017, 27.

[144] H. Cölfen, Macromol. Rapid Commun. 2001, 22, 219-252.

[145] M. Sedlák, H. Cölfen, Macromol. Chem. Phys. 2001, 202, 587-597 10.1002/1521-3935 (20010201) $202: 4<$ 587::AID-MACP587 > 3.0.CO;2-F.

[146] L. Qi, H. Cölfen, M. Antonietti, Angew. Chem. Int. Ed. 2000, 39, 604-607.

[147] T. Wang, A. W. Xu, H. Cölfen, Angew. Chem. Int. Ed. 2006, 45, 4451-4455.

[148] A. Ethirajan, U. Ziener, A. Chuvilin, U. Kaiser, H. Cölfen, K. Landfester, Adv. Funct. Mater. 2008, 18, 2221-2227.

[149] M. Helminger, B. Wu, T. Kollmann, D. Benke, D. Schwahn, V. Pipich, D. Faivre, D. Zahn, H. Cölfen, Adv. Funct. Mater. 2014, 24, 3187-3196.
[150] M. J. Olszta, X. Cheng, S. S. Jee, R. Kumar, Y.-Y. Kim, M. J. Kaufman, E. P. Douglas, L. B. Gower, Mater. Sci. Eng. R Rep. 2007, 58, 77-116.

[151] E. Ruiz-Hitzky, S. Letaïef, V. Prévot, Adv. Mater. 2002, 14, 439-443.

[152] C. E. Fowler, D. Khushalani, S. Mann, Chem. Commun. 2001, 2028-2029.

[153] M. Fernandez, K. Passalacqua, J. Arias, J. Arias, J. Struct. Biol. 2004, 148, 1-10.

[154] F. Nudelman, B. A. Gotliv, L. Addadi, S. Weiner, J. Struct. Biol. 2006, 153, 176-187.

[155] J. Perez-Carvajal, P. Aranda, A. Berenguer-Murcia, D. Cazorla-Amoros, J. n. Coronas, E. Ruiz-Hitzky, Langmuir 2013, 29, 7449-7455.

[156] L. Chen, S. Yang, E. Mäder, P.-C. Ma, Dalton Trans. 2014, 43, 12743-12753.

[157] P. Aranda, R. Kun, M. A. Martín-Luengo, S. Letaïef, I. Dékány, E. Ruiz-Hitzky, Chem. Mater. 2007, 20, 84-91.

[158] A. C. Alcântara, M. Darder, P. Aranda, E. Ruiz-Hitzky, Appl. Clay Sci. 2014, 96, 2-8.

[159] M. Darder, M. López-Blanco, P. Aranda, F. Leroux, E. RuizHitzky, Chem. Mater. 2005, 17, 1969-1977.

[160] F. M. Fernandes, I. Manjubala, E. Ruiz-Hitzky, Phys. Chem. Chem. Phys. 2011, 13, 4901-4910.

[161] C. Barrera, V. Florián-Algarin, A. Acevedo, C. Rinaldi, Soft Matter 2010, 6, 3662-3668.

[162] E. Ruiz-Hitzky, M. Darder, F. M. Fernandes, E. Zatile, F. J. Palomares, P. Aranda, Adv. Mater. 2011, 23, 5250-5255.

[163] J. Fripiat, M. Cruz-Cumplido, Annu. Rev. Earth Planet. Sci. 1974, 2, 239-256.

[164] H. Murray, Clay Miner. 1999, 34, 39-39.

[165] E. Ruiz-Hitzky, M. Darder, P. Aranda, J. Mater. Chem. 2005, 15, 3650-3662.

[166] E. Ruiz-Hitzky, P. Aranda, M. Darder, G. Rytwo, J. Mater. Chem. 2010, 20, 9306-9321.

[167] M. H. Delville, A. Taubert. Hybrid Organic-Inorganic Interfaces: Towards Advanced Functional Materials, John Wiley \& Sons, 2018.

[168] M. Darder, M. Colilla, E. Ruiz-Hitzky, Appl. Clay Sci. 2005, 28, 199-208.

[169] A. C. Alcântara, M. Darder, P. Aranda, S. Tateyama, M. K. Okajima, T. Kaneko, M. Ogawa, E. Ruiz-Hitzky, J. Mater. Chem. A 2014, 2, 1391-1399.

[170] Y. Koriche, M. Darder, P. Aranda, S. Semsari, E. Ruiz-Hitzky, Dalton Trans. 2014, 43, 10512-10520.

[171] M. Byun, N. B. Bowden, Z. Lin, Nano Lett. 2010, 10, 31113117.

[172] C. Beato, M. S. Fernández, S. Fermani, M. Reggi, A. NeiraCarrillo, A. Rao, G. Falini, J. L. Arias, CrystEngComm 2015, 17, 5953-5961.

[173] C. C. Tester, R. E. Brock, C.-H. Wu, M. R. Krejci, S. Weigand, D. Joester, CrystEngComm 2011, 13, 3975-3978.

[174] A. W. Xu, M. Antonietti, H. Cölfen, Y. P. Fang, Adv. Funct. Mater. 2006, 16, 903-908.

[175] F. Leroux, J.-P. Besse, Chem. Mater. 2001, 13, 3507-3515.

[176] G. O. Phillips, P. A. Williams, Gums and Stabilisers for the Food Industry 12, Royal Society of Chemistry, 2009. 
[177] M. Darder, A. Isabel Ruiz, P. Aranda, H. Van Damme, E. Ruiz-Hitzky, Curr. Nanosci. 2006, 2, 231-241.

[178] A. Bigi, S. Panzavolta, K. Rubini, Biomaterials 2004, 25, 5675-5680.

[179] A. Bajpai, N. Vishwakarma, J. Appl. Polym. Sci. 2005, 98, 42-52.

[180] A. I. Ruiz, M. Darder, P. Aranda, R. Jiménez, H. Van Damme, E. Ruiz-Hitzky, J. Nanosci. Nanotechnol. 2006, 6, $1602-1610$.

[181] A. K. Geim, K. S. Novoselov, Nat. Mater. 2007, 6, 183-191.

[182] K. S. Novoselov, A. K. Geim, S. V. Morozov, D. Jiang, Y. Zhang, S. V. Dubonos, I. V. Grigorieva, A. A. Firsov, Science 2004, 306, 666-669.
[183] M. Darder, E. Ruiz-Hitzky, J. Mater. Chem. 2005, 15, 39133918.

[184] A. Gómez-Avilés, M. Darder, P. Aranda, E. Ruiz-Hitzky, Angew. Chem. Int. Ed. 2007, 46, 923-925.

[185] T. Kyotani, T. Mori, A. Tomita, Chem. Mater. 1994, 6, 2138-2142.

[186] R. P. Feynman, Eng. Sci. 1960, 23, 22-36. 